NISTIR 7739

\title{
Open Monte Carlo Engine User Manual
}

Ruediger Kessel

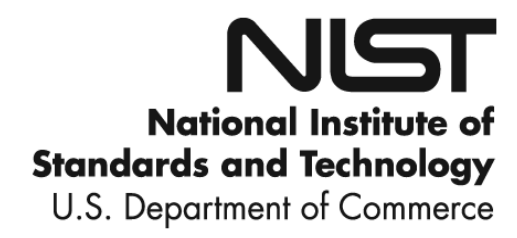


NISTIR 7739

\title{
Open Monte Carlo Engine User Manual
}

\author{
Ruediger Kessel \\ Information Technology Laboratory \\ Applied and Computational Mathematics Divisiont
}

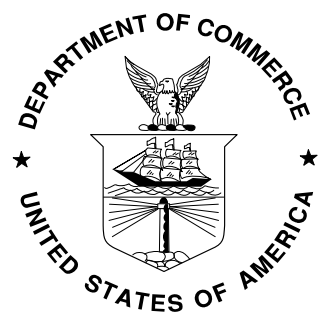

U.S. Department of Commerce Gary Locke, Secretary 


\section{Contents}

1 Introduction $\quad 3$

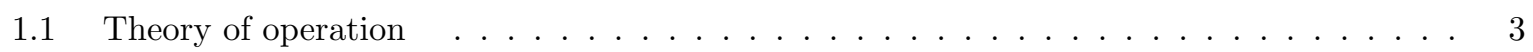

1.2 Limits and restrictions . . . . . . . . . . . . . . . . . . 3

1.3 Distribution of the $\mathrm{OMCE} \ldots \ldots \ldots \ldots \ldots$

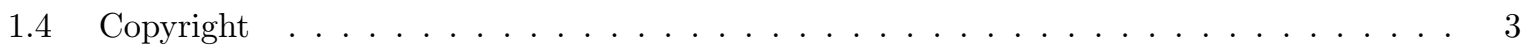

1.5 Disclaimer .............................. 3

2 Installing OMCE $\quad 4$

3 Running OMCE $\quad 4$

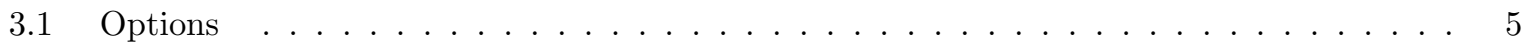

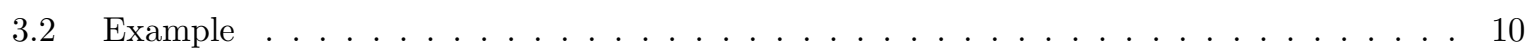

4 OMCE file formats $r$

$4.1 \mathrm{Xml}$ input file format . . . . . . . . . . . . . . . . . . . 10

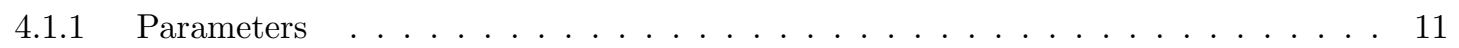

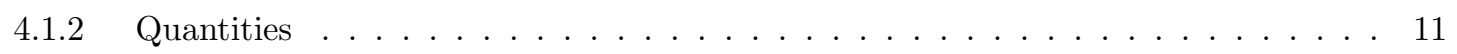

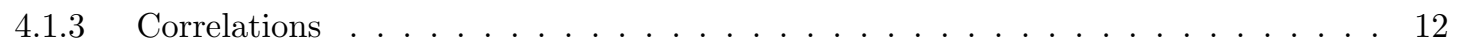

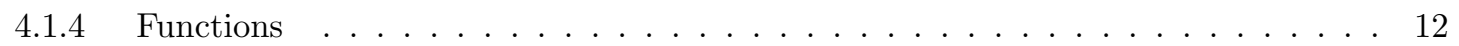

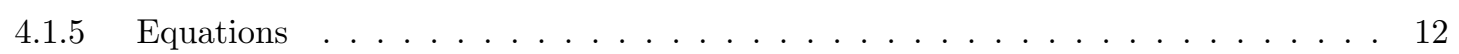

4.1 .6 Constraints . . . . . . . . . . . . . . . . . . . 12

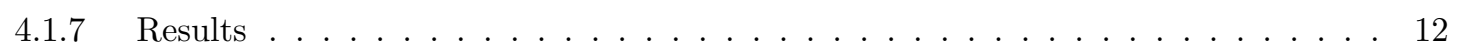

4.1 .8 Simulations .......................... 13

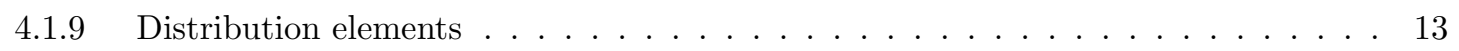

4.1.10 Expressions and equations ....................... 15

4.1 .11 Function minimum () and maximum ()$\quad \ldots \ldots \ldots \ldots$

4.1 .12 Function pow ()$\ldots \ldots \ldots \ldots$

4.1 .13 Function iff ()$\ldots \ldots \ldots \ldots \ldots \ldots$

4.1 .14 Logical expressions . . . . . . . . . . . . . . . . . . . . 16

4.1 .15 Symbol names . . . . . . . . . . . . . . . . . . . 17

4.2 Binary data file formats . . . . . . . . . . . . . . . . . 17

4.2.1 OMCE binary format . . . . . . . . . . . . . . . 17

4.2 .2 Simple binary format . . . . . . . . . . . . . . . . . 18

4.3 Text data file format . . . . . . . . . . . . . . . . . . . . . 19

$5 \quad$ Histogram viewer $r$

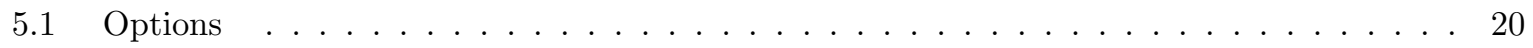

6 Adaptive mode $r$

6.1 GUM Supplement 1 based algorithm _. . . . . . . . . . . . . . . . . . . 20

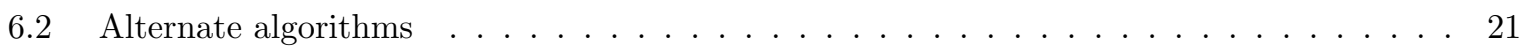




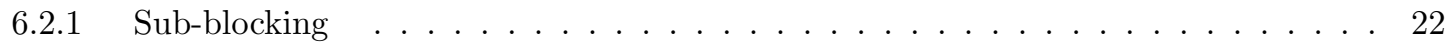

6.2.2 Establishing the statistical confidence level . . . . . . . . . . . . . . . . 22

$6.2 .3 \quad$ Numerical tolerance . . . . . . . . . . . . . . . . . . . . . . 22

6.2 .4 Predicting the number of simulation blocks $\ldots \ldots \ldots \ldots \ldots \ldots$

6.2 .5 Algorithms based on the Stein-method . . . . . . . . . . . . . . . 24

6.2.6 Algorithms based on a prediction of the needed runs . . . . . . . . . . . 24

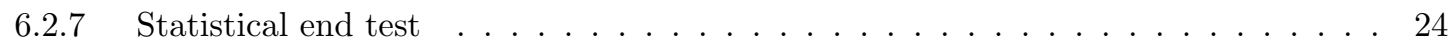

$7 \quad$ Running multiple simulations $\quad 25$

8 Input correlation coefficients $\quad 26$

9 Analysis of correlation $\quad 26$

10 Constraints on the domain of the functions 26

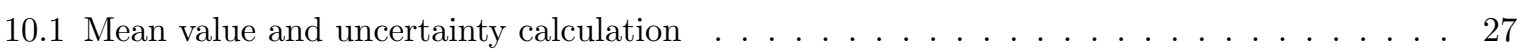

11 Testing and validation $\quad 28$

12 Performance 28

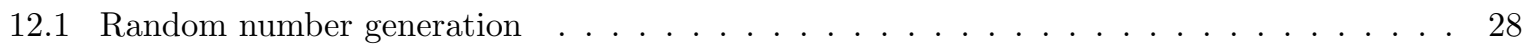

$\begin{array}{lr}\text { References } & 28\end{array}$

$\begin{array}{ll}\text { A Program exit codes } & 30\end{array}$ 


\section{Introduction}

The Open Monte Carlo Engine (OMCE) is a full featured command line Monte Carlo engine that reads a model description in xml-format and runs a Monte Carlo simulation to calculate a histogram, a best estimate (mean) and the standard uncertainty. In Addition, probabilistically symmetric or shortest coverage intervals are calculated. The OMCE can be used as an alternative method to the mainstream GUM [1] to evaluate the measurement uncertainty. The OMCE implements the Monte Carlo integration method as described in the Guide to the Expression of Uncertainty Supplement 1 [2]. The OMCE is written in Python and is public domain (open source code).

\section{$1.1 \quad$ Theory of operation}

The OMCE reads the model description in xml-format and creates an internal list of quantities, equations and results. It then evaluates the execution order for the equation system. A production and consumer pipeline is set up with two tasks. One task calculates a block of results and analyzes it, and the other task writes the block to a binary file and frees the memory. The pipeline stops when sufficient data are produced and completely written to the binary file. The last step is to write the histogram to a text file.

\subsection{Limits and restrictions}

The OMCE has the following limits and restrictions:

- The number of quantities, equations and results is not limited by the software. In practice the computer hardware, the memory and the computational power will limit the size of the model that can be simulated.

- Only explicit model equations can be simulated. OMCE does not include an equation solver.

\subsection{Distribution of the OMCE}

The OMCE is distributed as source code accompanied by an Windows executable (exe) file for the convenience of users who do not have Stackless Python [3] or Numpy [4] installed. The OMCE was developed with Stackless Python 2.5 but should run with other Stackless Python versions as well.

\subsection{Copyright}

The OMCE is free software with no copyright associated with it. It can be freely copied, modified and used, but it cannot be copyrighted. The idea is to have a Monte Carlo engine that can be freely studied and modified to user needs.

\subsection{Disclaimer}

This software was developed at the National Institute of Standards and Technology by employees of the Federal Government in the course of their official duties. Pursuant to title 17 Section 105 of the United States Code this software is not subject to copyright protection and is in the public domain. This software is experimental. NIST assumes no responsibility whatsoever for its use by other parties, and makes no guarantees, expressed or implied, about its quality, reliability, or any other characteristic. We would appreciate acknowledgement if the software is used. 


\section{Installing $\mathrm{OMCE}$}

OMCE is distributed as a zip-file (OMCE-1.1.14.zip) containing all necessary files to run it on a Windows platform without Python. The zip-file should be unpacked in a separate directory OMCE \. The OMCE can be executed directly from the command line if the OMCE \bin \subdirectory is added to the search path. The executable version (exe) contains all packages needed to run it. In addition, the source code is included. Linux users should use the source code version. The OMCE source code makes use of Stackless Python [3] and several non-standard open source Python packages imported in the beginning of OMCE.py. The packages must be installed before the source code version can be run.

\begin{tabular}{|c|c|c|}
\hline \multicolumn{3}{|c|}{ Contents of OMCE-1.1.14.zip } \\
\hline File & Location & Description \\
\hline OMCE.exe & OMCE $\backslash \mathrm{bin} \backslash$ & Exe version of the OMCE \\
\hline OMCE.xsd & OMCE $\backslash \mathrm{bin} \backslash$ & xml schema definition of the OMCE format \\
\hline OMCE. ofd & $\mathrm{OMCE} \backslash \mathrm{bin} \backslash$ & OMCE statistical output file definition \\
\hline OMCE.vfd & $\mathrm{OMCE} \backslash \mathrm{bin} \backslash$ & OMCE var file definition \\
\hline ALT. of d & $O M C E \backslash b i n \backslash$ & alternative statistical output file definition \\
\hline ALT.vfd & OMCE $\backslash \mathrm{bin} \backslash$ & alternative var file definition \\
\hline$* . *$ & OMCE $\backslash \mathrm{bin} \backslash$ & python runtime environment \\
\hline OMCE.pdf & $\mathrm{OMCE} \backslash \mathrm{doc} \backslash$ & OMCE user manual \\
\hline OMCE \Schema.html & $\mathrm{OMCE} \backslash \mathrm{doc} \backslash$ & OMCE xml schema description \\
\hline OMCE.py & OMCE\src $\backslash$ & OMCE Python source file \\
\hline OMCE.Xsd & OMCE $\backslash s r c \backslash$ & xml schema definition of the OMCE format \\
\hline OMCE. of d & $O M C E \backslash \operatorname{src} \backslash$ & OMCE statistical output file definition \\
\hline OMCE.vfd & OMCE $\backslash s r c \backslash$ & OMCE var file definition \\
\hline ALT. of d & OMCE $\backslash s r c \backslash$ & alternative statistical output file definition \\
\hline ALT.vfd & OMCE\src $\backslash$ & alternative var file definition \\
\hline Viewer.py & $\mathrm{OMCE} \backslash \mathrm{src} \backslash$ & histogram viewer \\
\hline $\begin{array}{l}\text { Viewer.exe } \\
* . *\end{array}$ & $\begin{array}{l}\text { OMCE } \backslash \text { Viewer } \backslash \\
\text { OMCE } \backslash \text { Viewer } \backslash\end{array}$ & $\begin{array}{l}\text { EXE-version of the histogram viewer } \\
\text { python run-time environment for the viewer }\end{array}$ \\
\hline *. omc & OMCE\examples \} $&{\text { example xml files in OMCE format }} \\
{\hline * . \mathrm{xml}} &{\text { OMCE\examples \ }} &{\text { example xml files in alternativee format }} \\
{\hline \text { !run_all_OMCE. bat }} &{\text { OMCE \examples \ }} &{\text { batch file to run all examples in OMCE format }} \\
{\hline \text { !run_all_ALT.bat }} &{\text { OMCE \examples } \backslash} &{\text { batch file to run all examples in alternative format }} \\
{\hline \text { *. ohd }} &{\text { OMCE \examples \ref } \backslash} &{\begin{array}{l}\text { reference results for the examples in OMCE format } \\
(- \text { seed }=1)\end{array}} \\
{\hline * \text {. sta }} &{\text { OMCE } \backslash \text { examples } \backslash r e f \backslash} &{\begin{array}{l}\text { reference results for the examples in alternative for- } \\
\text { mat }(- \text { seed }=1)\end{array}} \\
$\hline
\end{tabular}

After the installation, the windows batch file !run_all_OMCE.bat or !run_all_ALT.bat in OMCE\examples should be run to verify the installation. This will run the example xml files and compare the results with the reference results stored in OMCE \examples $\backslash r$ ef $\backslash$. The random number generator is seeded for these runs so the results must exactly match the reference results.

\section{Running OMCE}

The OMCE is a command line tool which is designed to be used in batch scripts or to be called by other programs. If the directory of OMCE (exe-version) is included in the path, it can be executed with the following command:

OMCE filename.omc

This will start the OMCE, loading filename.omc and creating a file named filename.ohd. All options have default values. If no file name is given, a list of options with their default values and the implemented error codes is printed. 
The general format for using the OMCE is:

OMCE <filename.omc $>\mid<$ filename.xml> [<options $>$ [<parameters $>]$

The input file should be either in omc-format or in the alternative xml-format. See option $-f i$ and Section 4.1 on file formats for more details.

All option follow the following format:

-<option $>=<$ value $>$

The option name <option> is string of one to four characters identifying the option and is always followed by an equal sign. The format of the <value $>$ depends on the option and can be either an integer, a floating point number or a string. See Section 3.1 for a detailed discussion.

Parameters have the following format:

$<$ name $>=<$ value $>$

The parameter names are defined in the omc input file (see Section 4.1.1). The value must be a floating point number.

Note: The name of the input file name must be specified before any parameters.

If there is an error, OMCE will terminate with a non-zero exit code. See Appendix A for a list of possible error messages and exit codes.

\section{$3.1 \quad$ Options}

The options can be given in any order but they should be separated by a space and should be given after the omc-file name.

-a This switch activates the generation of the statistical data file (.ohd) and is set to 1 by default. This switch should be set to zero if the internal data analyzer is not used and the binary data file $(-w b=1$ or $-w b=2)$ is analyzed by some external tool.

-ac This option controls the correlation analysis of the simulated output data.

\begin{tabular}{|c|l|}
\hline$-\mathrm{ac}=$ & correlation analysis \\
\hline 0 & no correlation analysis \\
1 & between results (default) \\
2 & results and their inputs \\
3 & all \\
\hline
\end{tabular}

-ad This option sets the adaptive mode tolerance divisor to the value given (see section on adaptive mode). The tolerance is calculated based on the standard uncertainty or the probability interval and the number of significant digits divided by the tolerance divisor. The default value is 1.0 .

The adaptive mode is controlled by the following options: -ad, -af, -am, -ap, -at, -k, -mr, - sd.

-af This option sets the probability level for the decisions during the adaptive mode. If option $-\mathrm{k}=0$ or if the alternate algorithms are used $(-\mathrm{am}>0)$ a $t$-factor is calculated based on the probability controlled by this option and the number of simulation runs so far.

The adaptive mode is controlled by the following options: -ad, -af, -am, -ap, -at, -k, -mr, - sd.

-am This option controls the method how the number of runs are determined during the adaptive mode. The default $-a m=0$ uses the sequential checking scheme defined in GUM Supplement 1. Alternatively, - am $>0$ activates alternative 2-stage schemes to predict the total number of runs. 


\begin{tabular}{|c|l|}
\hline- am $=$ & adaptive algorithm used during simulation \\
\hline 0 & GUM Supplement 1 based algorithm \\
1 & modified Stein 2-Stage scheme (see [5]) with S1 accuracy definition \\
2 & modified Stein 2-Stage scheme (see [5]) with fixed relative accuracy definition \\
3 & prediction of the needed runs with S1 accuracy definition \\
4 & prediction of the needed runs with fixed relative accuracy definition \\
\hline
\end{tabular}

The adaptive mode is controlled by the following options: -ad, -af, -am, -ap, -at, -k, -mr, - sd.

-ap This option sets the probability of the interval which is used for the tolerance check in the adaptive mode. This interval is calculated for every block of data. If bit 2 of the option -at is set then the standard deviation of the average of all calculated intervals is multiplied by the $k$-factor (option -k) and compared with the tolerance based on option - sd and -ad.

The adaptive mode is controlled by the following options: -ad, -af, -am, -ap, -at, -k, -mr, - sd.

This option controls in detail which parameter is used to for the check in the adaptive mode (see Section 6). The value of this option is a bit combination which enables the different checks The default is to the check the standard deviation of the mean, the standard uncertainty and the limits.

\begin{tabular}{|c|l|}
\hline- at $=$ & parameter used during the check \\
\hline 1 & standard deviation of the means \\
2 & standard deviation of the uncertainty \\
3 & standard deviation of the means and the uncertainty \\
& (see [2] Section 7.9.4 Note 5) \\
standard deviation of the means, the uncertainty and the limits & (default see [2] Section 7.9.4) \\
\hline
\end{tabular}

-bs The internal data processing in the OMCE operates on data blocks with a fixed block size. The block size has influence on the performance and the statistical properties of the result. Usually the default block size (10000 values) is a good compromise between performance and statistical rigor. The block size can be changed for experimental purposes or if the value of option $-\mathrm{nl}$ or $-\mathrm{nc}$ is reduced.

In the adaptive mode the block size is adjusted automatically and the value given with this option will be overridden.

-c1 This option controls the lower limit of the absolute value of the calculated correlation coefficient. The default value is 0.001 .

-da By default $(-\mathrm{da}=0)$ an existing output file will be replaced by new simulation data. If this option is set to $-\mathrm{da}=1$, no header is printed to the file and the data is appended. If the data should be distinguishable from prior existing data, set the option -sid to a different start value. Warning: The format of the existing output file will not be checked.

-de This option controls how the name of the output files is formed from the given input filename. If this option is set (default), the extension (.omc) is deleted from the filename before the output file extension is added; otherwise the extension is added without deletion. Warning: any existing output file (.dat, .bin, .ohd, .sta) will be overwritten without warning.

-e1 The OMCE will automatically try to correct for small negative eigenvalues in the correlation matrix. This option controls the limit for the correction. The value must be negative and the default is -0.1 .

-e2 When the correlation matrix needs to be corrected, the Least Maximum Norm of the correction is calculated. This option controls the limit for this norm. The default value is 0.01 . 
-fi The OMCE supports two input formats which are both based on xml. The native OMCE format allows a simple description of the evaluation model. The details of the format can be found in OMCE_schema.html. As an alternative, OMCE can read an alternative xml-format.

\begin{tabular}{|c|l|}
\hline$-f i=$ & input format \\
\hline 0 & OMCE format (default) \\
1 & alternative xml-format \\
\hline
\end{tabular}

-fo As a default, OMCE forms the output file name based on the given input file name. With this option an explicit output file name can be given.

-hc The resolution of the histogram is set by this option (number of bars). The value should be between 1 and 100000. The default is 200 bars.

-hf The width of the histogram is extended by the factor given with this option. The value should be between 0.1 and 100.0 . The default is 1.2 .

-hp The width of the histogram is controlled by a probability interval to capture the relevant part of the distribution. The probability of the interval should be given as a value between 0.001 and 1 . A value of 1 creates an interval that contains all values calculated during the simulation. The default is $0.99(99 \%)$.

- $\mathbf{i}$ This options controls the interpretation of the coverage intervals. By default $(-i=0)$, all coverage intervals are probabilistically symmetric. If the option is non-zero then the shortest coverage intervals are calculated.

-info If the info option is non zero, OMCE prints details about the xml input file or some internal tables and quits.

\begin{tabular}{|c|l|}
\hline -info $=$ & description \\
\hline 0 & (no information) \\
1 & $\begin{array}{l}\text { details of the model given in the xml-file: } \\
\text { input quantities } \\
\text { equations }\end{array}$ \\
& $\quad \begin{array}{l}\text { execution order } \\
\text { results }\end{array}$ \\
& list of predefined symbols and functions \\
3 & list of symbols usable in format definition files \\
4 & list of reserved words not to be used as quantity names \\
5 & list of error messages in LaTeX format \\
\hline
\end{tabular}

-io This option controls the sampling for imported quantities (see element <Import> in Section 4.1.9). If $-i o=0$ (default) then the binary data is sampled at random from binary files. If $-i o=1$ then the data is sampled sequentially from the binary file in the same (reproducible) order as it was written. If the number of runs (-mcs) is equal to the number of values in the binary file then the exact same sequence for the quantities is reproduced.

-k The k-factor scales the tolerance check during the adaptive mode. The default value is 2.0. A larger value will require more Monte Carlo runs. If this option is set to zero, the k-factor will be evaluated from a $t$-table (see option -ap) look up based on the number of runs so far.

The adaptive mode is controlled by the following options: -ad, -af, -am, -ap, -at, -k, -mr, - sd.

-1 This option allows to repeat the simulation the given number of times is it is set not equal to zero. Hint: This option is only effective if the omc-file does not contain a section $<$ Simulations $>$ and will be ignored otherwise. The default value is $-1=0$.

-lc The correlation matrices will be printed if this option is set. The default is $-1 \mathrm{c}=0$. 


\begin{tabular}{|c|l|}
\hline$-l c=$ & description \\
\hline 0 & no matrix printed (default) \\
1 & print input correlation matrix \\
2 & print result correlation matrix \\
3 & print input and result correlation matrix \\
\hline
\end{tabular}

- Imn This option defines the matrix norm type.

\begin{tabular}{|c|l|}
\hline$-1 m n=$ & description \\
\hline 0 & plain norm \\
1 & weighted norm (default) \\
\hline
\end{tabular}

The weighted norm uses the larger correlation coefficient as a weight for the difference. The minimum weight is 0.1 .

-lt If this option is set $(-1 t=1)$, OMCE prints the tolerances used in the adaptive mode together with the standard deviations evaluated (see section on adaptive mode for details).

-mcp This option selects the matrix correction procedure for the the correlation matrix in the event that the matrix is found to be not positive semi-definite.

\begin{tabular}{|c|l|}
\hline$-\mathrm{mcp}=$ & description \\
\hline 0 & spectral decomposition (default) \\
1 & Near4 (N. J. Higham [6]) \\
2 & Random Walk \\
\hline
\end{tabular}

-mcs This option controls the number of simulation blocks. The total number of trails is the value of this option multiplied by the block size (option -bs). This option will override the value given in the xml-file. A value of zero or minus one activates the adaptive mode. The default value is -1 .

$-\mathrm{mr} \quad$ To prevent the simulation from running for an excessive amount of time during the adaptive mode, a maximum number of blocks (runs) can be set by this option. The value given here is multiplied by the block size (see option -bs). The default value is 10000 blocks.

The adaptive mode is controlled by the following options: -ad, -af, -am, -ap, -at, -k, -mr, - sd.

This option controls the fraction of valid data that is needed to evaluate the correlation coefficients. The default value is $-n l=0.5$. The simulation is aborted if the fraction of valid data available to calculate the correlation coefficients in one data block is smaller than the value controlled by this option. Lowering this value might only be advisable if the block size is increased at the same time.

-nl This option controls the fraction of valid data that is needed to continue the simulation. The default value is $-\mathrm{nl}=0.5$. The simulation is aborted if the fraction of valid data in one data block is smaller than the value controlled by this option. Lowering this value might only be advisable if the block size is increased at the same time.

-ofd This option controls the file name for the output format definition file. The definition file should be placed in the same directory as the OMCE executable or python source. The default is 'OMCE'.

-p1 This option controls the exact probability of the $95 \%$ interval. The default is $-\mathrm{p} 1=0.95$.

-p2 This option controls the exact probability of the $99 \%$ interval. The default is $-\mathrm{p} 2=0.99$.

-p3 This option controls the exact probability of the $99.9 \%$ interval. The default is $-\mathrm{p} 3=0.999$.

-pa This option controls the exponent used for the calculation of the average of the quantiles. If $a$ is the value set by this option then the average is calculated based on the equation

$$
\bar{q}=\sqrt[a]{\frac{1}{n} \sum_{i=1}^{n} q_{i}^{a}}
$$


The default value is $-\mathrm{pa}=2.0$

-pdf The data in the histogram can either be counts $(-\mathrm{pdf}=0)$ or samples of a PDF (probability density function) (-pdf=1). The PDF samples are useful if the data is to be compared with a given PDF. The default is $-p d f=1$.

-po If this option is given, OMCE will print a list of all effective options and quits. This option is useful for documenting and debugging the command line options.

-pq This option controls the quantile estimation method. The details to the estimation methods can be found in [7].

\begin{tabular}{|c|l|}
\hline$-\operatorname{lmn}=$ & description \\
\hline 0 & no interpolation (see $[2]$ Section 7.7.2) (default) \\
1 & linear interpolation at $n_{\mathrm{b}} \times p+1 / 2(\mathrm{R}-5)$ \\
2 & linear interpolation at $\left(n_{\mathrm{b}}+1 / 3\right) p+1 / 3(\mathrm{R}-8)$ \\
3 & linear interpolation at $\left(n_{\mathrm{b}}+1 / 4\right) p+3 / 8(\mathrm{R}-9)$ \\
4 & linear interpolation at $\left(n_{\mathrm{b}}+2\right) p+1 / 2$ \\
\hline
\end{tabular}

-pr The simulation is done in two steps. At the start a number of runs controlled by this option will be executed. After the first runs are completed, the binning interval and the tolerance for the adaptive mode are calculated and these values are used during the rest of the simulation runs. The default is 10 first runs.

-sbs This option controls the sub block size for the alternate adaptive mode algorithms. The sub block mechanism is not activated by default $(-\mathrm{sbs}=-1)$. The minimum sub block size is 100 . The block size must be devidable by the sub block size.

The adaptive mode is controlled by the following options: -ad, -af, -am, -ap, -at, -k, -mr, - sd.

-sd This option controls the number of significant digits for the adaptive mode. The default is 2 digits $(-s d=2)$.

The adaptive mode is controlled by the following options: -ad, -af, -am, -ap, -at, -k, -mr, - sd.

-seed The seed of the random number generator can be set to a fixed value given by this option. A default value of zero will force a random seeding based on the system clock.

-sev This options controls the value of the smallest eigenvalue after correction. The default is $1 \mathrm{E}-7$.

-sid This option controls the start value for the simulation ID. The default value is 0 . The simulation ID will be incremented after every simulation.

-t The OMCE will measure and print the time elapsed to run a complete simulation if $-\mathrm{t}=1$. More detailed information on the time elapsed for the omc-file reading, simulation and the ohd-file creation will be printed if $-\mathrm{t}=2$.

-v This option controls the verbose level between 0 for all messages and 10 for no messages. The default value is 2 .

\begin{tabular}{|c|l|}
\hline$-\mathrm{v}=$ & description \\
\hline 9 & error messages \\
8 & warnings \\
7 & startup message \\
6 & finish message \\
5 & simulation progress \\
4 & timing information \\
3 & standard information \\
2 & default value \\
1 & additional information and calculation details \\
0 & everything else \\
\hline
\end{tabular}


Note: information requested by other options (like -info, etc.) will be suppressed if the verbose level is set larger as 3 .

-vfd This option gives the file name for the var-file format definition file. The definition file should be placed in the same directory as the OMCE executable or the Python source code. The default is 'OMCE'.

-vm This option activates the validation mode if it is set. During validation mode all simulation data are kept in memory and are analysed when the simulation runs are complete.

-wb The binary data calculated during the simulation can be written to a binary data file. This option controls the generation and the format. For the sorted format $(-w b=3)$ the binary data will be sorted and made monotonic prior writing to the file. The option $-w b=3$ should not be used if the data will be imported in other simulations via the element <Import>.

\begin{tabular}{|c|l|}
\hline$-\mathrm{wb}=$ & format description \\
\hline 0 & no binary data file \\
1 & OMCE format \\
2 & simple format \\
3 & sorted OMCE format \\
\hline
\end{tabular}

The default is $-\mathrm{wb}=0$. See the section on binary data format for details.

-wv This option enables the creation of a .var output file. By Default no .var file is generated $(-\mathrm{wV}=0)$.

-xsd The OMCE can validate the xml-input file against an xsd-file. With this option, the path to the xsd-file can be given. By default, the option is '-' and OMCE.xsd is used for omc-files and no validation is done for alternative xml-files. (see option $-\mathrm{fi}$ ).

\subsection{Example}

OMCE S02. omc $-\mathrm{pdf}=1-\mathrm{hc}=400-\mathrm{t}=1$

The OMCE will load the file S02.omc from the current directory and it will create the file S02.ohd containing histogram data with a resolution of 400 bins converted to a PDF sampling. The whole operation will be timed and the elapsed time will be shown.

\section{OMCE file formats}

The OMCE accepts two different xml-file formats discussed in the section about xml input formats. The OMCE creates data files of different types. The raw data is written to binary files and the histogram data and the other information is written to ASCII files in comma separated value format. The extension is .ohd.

\subsection{Xml input file format}

The native OMCE input file format (extension omc) is a simple xml-format. It describes the model of evaluation and the information about the input quantities. See OMCE \doc \OMCE_Schema.html for details about the omc-file format structure. An omc-file has the following basic structure:

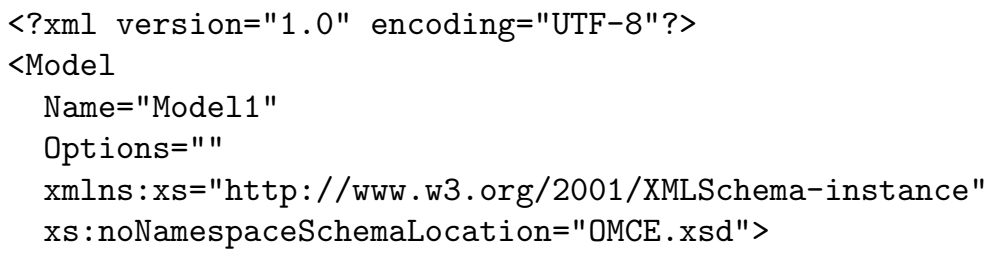




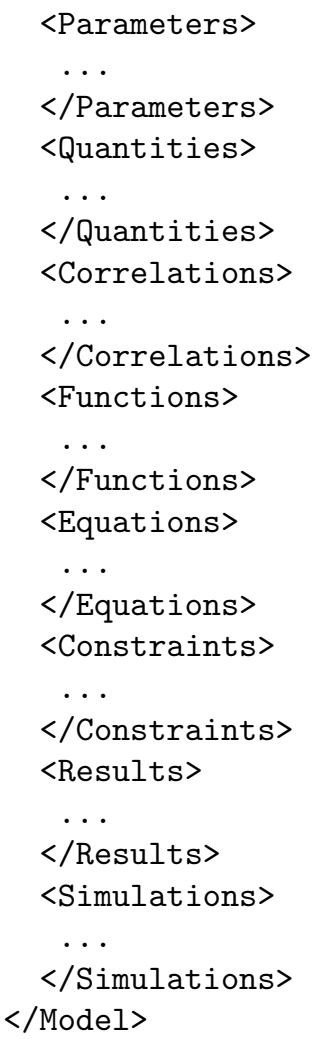

A <Model> element contains all information about the evaluation model. The Name and the Options attributes are optional. The Options attribute may contain a list of space separated command line options. The information about the schema is optional and is not used in OMCE. The validation schema is controlled by the option -xsd.

\subsubsection{Parameters}

The optional section <Parameters > defines a list of symbolic model parameter values that can be used in the parametric specifications of the quantities. In this section the default values of the parameters are specified. A parameter specification has the following structure:

$<$ Parameter Name="P" Value="1.0"/>

All attributes are mandatory. The attribute Name defines the parameter name (e.g. "P") by which the parameter is referred to in the definition of the quantities. The attribute Value assigns the default value to the parameter. The value of the parameter can be re-assigned by a command line parameter (see Section 3) or inside the section <Simulations $>$.

\subsubsection{Quantities}

The section <Quantities> defines a list of quantities which can be used in the model equations. A quantity has the following structure:

<Quantity Symbol="X" Unit="kg"> .. </Quantity $>$

The attribute Symbol is mandatory and defines the symbol name (e.g. "X") by which the quantity is referred to in the equations. The attribute Unit is optional. A quantity element should contain one distribution element. 


\subsubsection{Correlations}

The section <Correlations> is optional and defines a list of correlation coefficients which are used during the simulation runs. A correlation coefficient has the following structure:

<Coefficient Q1="X_1" Q2="X_2" Value="0.5" />

All attributes are mandatory. Attribute Q1 and Q2 define the quantities for the correlation coefficient and Value defines its value. The value must be greater than or equal to -1 and smaller than or equal to +1 . All quantities where a correlation coefficient is given must be normal distributed. All correlation coefficients together must form a matrix which is positive semi-definite.

\subsubsection{Functions}

The section <Functions> is optional and defines a list of user defined functions to be used in <Equation> and <Result $>$ elements. A function definition has the following structure:

<Function Symbol="f" Parameter="p1,p2" Global="X_1,X_2"> . . </Function>

The attribute Symbol is mandatory and defines the function name (e.g. " $\mathrm{f}$ ") by which the function is referred to in <Equation> and <Result $>$ elements. The optional attribute Parameter defines a parameter list for the function. The optional attribute Global defines a list of quantities or equation names that can be used inside the function in addition to the given parameters. The element <Function> should contain a valid expression.

\subsubsection{Equations}

The section <Equations> defines a list of equations which are evaluated during the simulation runs. A equation has the following structure:

$<$ Equ Symbol="Y" Unit="kg"> .. </Equ $>$

The attribute Symbol is mandatory and defines the symbol name (e.g. "X") by which the equation is referred to in the result list or in other equations. Every equation must have a unique symbol name. The attribute Unit is optional. An element <Equ> should contain an expression which can be evaluated.

\subsubsection{Constraints}

The section <Constraints> defines a list of constraints which need to be met by the data during the simulation. A constraint has the following structure:

<Constraint $\mathrm{Q}=" \mathrm{X} ">\ldots</$ Constraint $>$

The attribute $\mathrm{Q}$ is mandatory and defines the name of the quantity (e.g. "X") referencing the data which is validated by the constraint. An element <Constraint> should contain a logical expression which can be evaluated to True or False. Logical expressions for constraints on input quantities can only refer to input quantities while logical expressions for constraints on quantities defined by equations can refer to any quantities. Quantity names referring to results cannot be used with constraints.

\subsubsection{Results}

The section <Results $>$ defines a list of results which are observed during the simulation runs. A result has the following structure:

$<$ Result Symbol="Y" $>$ Y $</$ Result $>$ 
The attribute Symbol is mandatory and defines the symbol name (e.g. "Y") by which the result is referred to in the ohd- or var-file. The same symbol name may be used for both a result and an equation. A result element should contain a valid expression. Usually this expression contains only the name of an equation. Result symbols cannot be used in expressions for equations or results.

\subsubsection{Simulations}

The optional section <Simulations> provides a simple tool to define multiple simulations which are sequentially executed. If the section is not present in an omc-file, only one simulation is executed. All the result data for the simulations is written to the same file. The section <Simulations $>$ contains a list of the elements <Simulation> and <Loop> given in any order.

The element <Simulation> defines one simulation run and it has the following structure:

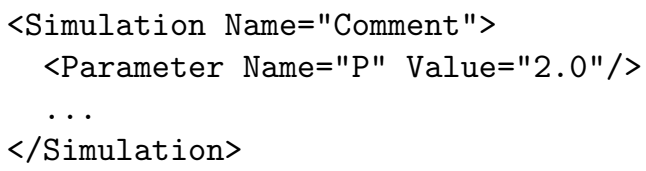

The attribute Name is optional and it specifies a comment that will be printed when the simulation is executed. The element <Simulation> can contain a list of parameter re-assignments. All parameter must be defined in the section <Parameters $>$ before they can be used here.

The element <Loop> defines a for-loop for multiple simulations and it has the following structure:

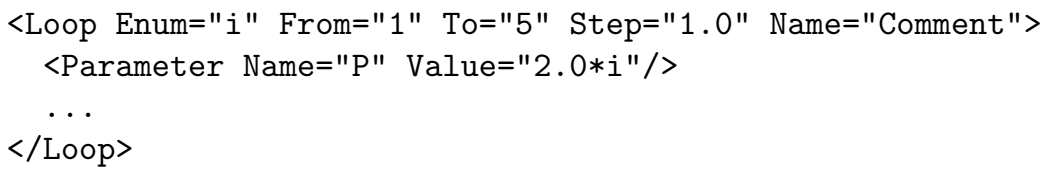

The attribute Enum is optional and specifies a name for the enumerator. If the attribute is present the name given in Enum can be used to evaluate the values of the parameters.

The attributes From and To are mandatory and they define a range for the loop enumerator. The optional attribute Step specifies the value of the increment that is added (or subtracted) to the enumerator after every simulation. The default value for the Step is 1.0.

Note: The loop will always start with a value for the enumerator given in From independent if the value given in To is smaller or larger than From. The given sign of Step is ignored and the sign is adjusted so that the numerator is changed after every run in the direction of To.

The attribute Name is optional and specifies a comment that will be printed when the simulation is executed.

\subsubsection{Distribution elements}

A distribution element defines the information which is available about the quantity. The following distribution elements can be used inside a quantity element:

$\langle$ Constant $\rangle\langle$ Value $>1.0</$ Value $\rangle</$ Constant $\rangle$

The element <Constant> defines a constant quantity without uncertainty.

$<$ Normal $><$ Mean $>0.0</$ Mean $>\langle$ Sigma $>1.0</$ Sigma $></$ Normal $>$

The element <Normal $>$ defines a normally distributed quantity with the specified mean value and the specified standard deviation (Sigma).

$\langle$ Student $>\langle$ Mean $>0.0</$ Mean $>\langle$ Scalefactor $>1.0</$ Scalefactor $>\langle$ Dof $>5</$ Dof $\rangle\langle/$ Student $\rangle$ The element $<$ Student $>$ defines a student-t distributed quantity with the specified mean value and degrees of freedom (Dof) scaled by the specified scaling factor (Scalefactor). 
<Rectangle > Mean>0.0</Mean> <Halfwidth $>1.0</$ Halfwidth $></$ Rectangle $>$

The element $<$ Rectangle $>$ defines a rectangularly distributed quantity with the specified mean value and the specified half-width (semi-width).

<Triangle> <Mean>0.0</Mean> <Halfwidth>1.0</Halfwidth> </Triangle>

The element $\langle$ Triangle $>$ defines a triangularly distributed quantity with the specified mean value and the specified half-width (semi-width).

<Trapez> <Mean>0.0</Mean> <Halfwidth>1.0</Halfwidth> <Beta>0.5</Beta> </Trapez>

The element $<$ Trapez $>$ defines a trapezoidal distributed quantity with the specified mean value and the specified halfwidth (semi-width). Beta is the ratio between the evenly distributed part and the width of the distribution.

$\langle$ Ctrapez $\rangle\langle$ Mean $>0.0</$ Mean $\rangle\langle$ Halfwidth $>1.0</$ Halfwidth $\rangle\langle$ Beta $>0.5</$ Beta $\rangle\langle/$ Ctrapez $\rangle$

The element $<$ Ctrapez $>$ defines a iso-curvilinear trapezoidal distributed quantity [8] with the specified mean value and the specified half-width (semi-width). Beta is the ratio between the evenly distributed part and the width of the distribution.

<Ushape> <Mean>0.0</Mean> <Halfwidth>1.0</Halfwidth> </Ushape>

The element $<$ Ushape $>$ defines a U-shaped (arcus-sinus) distributed quantity with the specified mean value and the specified half-width (semi-width).

$\langle$ TypeA $>\langle$ Value $>1.0</$ Value $>\ldots \quad\langle/$ TypeA $>$

The element $<$ TypeA $>$ defines an observed quantity. It contains at least $2<$ Value $>$ elements.

<Poisson> <Mean>1.0</Mean> </Poisson>

The element $<$ Poisson $>$ defines a Poisson distributed quantity with the specified mean value.

<Exponent> <Mean>1.0</Mean> </Exponent>

The element $<$ Exponent $>$ defines a exponentially distributed quantity with the specified mean value.

$\langle$ Gamma $\rangle\langle$ Shape $>1.0</$ Shape $\rangle\langle$ Scale $>1.0</$ Scale $\rangle\langle/$ Gamma $\rangle$

The element $<$ Gamma $>$ defines a Gamma distributed quantity with the specified shape and scale value.

<Discrete> <Binfile Filename="filename.bin" Index="O" Q="Y"/> </Discrete>

The element $\langle$ Discrete $>$ defines a discrete distribution based on binary data (see [2] Section 7.5.1). The element $\langle$ Discrete $>$ contains the mandatory element $\langle$ Binfile $>$ defining the binary data source. The binary data is read from a file given by the attribute Filename. The file should exist and it should have a valid binary format (see option -wb). The path should be either relative to the current XML-file or an absolute file path.

Binary files can contain binary data for multiple quantities. The optional attribute Index defines the index of the quantity in the binary file. The first quantity is the default and has the index 0 . If the binary contains a symbol table the optional attribute $Q$ can specify the name of the quantity instead of its index. The attribute Index will be ignored if an attribute $\mathrm{Q}$ is specified.

After the element <Binfile> two optional elements <Shift> and <Scale> can be used to define a scale value all data is multiplied with and a shift value which is added to all (shifted) values. Multiple quantities might share the same binary data with different shift and scale values. Although the these quantities share the same data, they are uncorrelated.

<Import> <Binfile Filename="filename.bin" Index="0" $Q=" Y " /></$ Import>

The element <Import> is similar to the element <Discrete>. It contains only the element $\langle$ Binfile $\rangle$. No other optional elements are allowed. See the paragraph above about $\langle$ Discrete $\rangle$ for details about the element $\langle$ Binfile $\rangle$. As long as only one quantity is imported per file there is virtually no difference between element <Discrete> and element <Import>. The user might use the the element <Discrete> if the distribution needs to be scaled or shifted. 
If multiple quantities are imported from the same file with the element <Import> then the dependency structure (correlation) present in the binary data will be maintained in the current simulation by sampling jointly for all quantities from this file. Option -io controls if a random sampling $(-i o=0)$ or a sequential sampling $(-i o=1)$ is used.

The same binary file can be used for a <Discrete $>$ element and for a < Import $>$ element by using the same filenames and indices. The quantities share the distribution but they are sampled independently (not correlated).

\subsubsection{Expressions and equations}

The elements <Equ>, <Function> and <Result> require an expression as their content. The format of the expression is basically the format of a Python expression. The operator $+,-, *, /$ and $* *$ can be used in expressions together with predefined functions, constants and the parentheses. The option - info=2 lists the predefined functions and constants.

\begin{tabular}{|c|l|l|}
\hline \multicolumn{2}{|c|}{ Predefined functions } \\
\hline Function & Description & Remarks \\
\hline abs() & Absolute value, Modulus & \\
$\operatorname{acos}()$ & Arc cosine & \\
$\operatorname{asin}()$ & Arc sine & \\
$\operatorname{atan}()$ & Arc tangent & \\
$\cos ()$ & Cosine & \\
$\cosh ()$ & Hyperbolic cosine & see Section 4.1 .13 \\
$\exp ()$ & Natural exponential function (base e) \\
iff() & Value selection & \\
$\log ()$ & Natural logarithm (base e) & see Section 4.1.11 \\
$\log 10()$ & Common logarithm (base 10) & see Section 4.1 .11 \\
$\max ()$ & Maximum value & see Section 4.1 .11 \\
$\operatorname{maximum()}$ & Maximum value & see Section 4.1 .11 \\
$\min ()$ & Minimum value & see Section 4.1 .12 \\
$\operatorname{minimum()}$ & Minimum value & \\
$\operatorname{pow}()$ & Power & \\
$\sin ()$ & Sine & \\
$\sinh ()$ & Hyperbolic sine & \\
$\operatorname{sqr}()$ & Square & \\
$\operatorname{sqrt()}$ & Square root & \\
$\tan ()$ & Tangent & \\
$\tanh ()$ & Hyperbolic tangent & \\
\hline
\end{tabular}

\begin{tabular}{|c|l|l|}
\hline \multicolumn{3}{|c|}{ Predefined constants } \\
\hline Constant & Description & Value \\
\hline e & Euler's number & 2.7182818284590451 \\
pi & number pi & 3.1415926535897931 \\
\hline
\end{tabular}

The predefined functions and constants are a direct mapping of the NumPy functions with the same name. For details consult the NumPy documentation [4]. Most of the functions except minimum(), $\min (), \operatorname{maximum}(), \max ()$, pow() and iff() have one argument. All function return one result.

OMCE allows to use distribution functions in <Equ>, <Function> and <Result> elements. These functions are useful to model statistical effects without assigning a quantity name to it. This is particular useful in function definitions to create certain statistically independent properties every time the function is used. All parameters of the distribution functions must evaluate to constant values by either being a numerical constant or an arithmetic expression using only quantities which are defined as constants or by using the quantity access functions val() or $\mathrm{u}($ ) (see below). 


\begin{tabular}{|c|l|l|}
\hline \multicolumn{2}{|c|}{ Predefined distribution functions } \\
\hline Function & Description & Parameter \\
\hline Rectangle() & Rectangular distribution & Mean value, half-width \\
Normal() & Normal distribution & Mean value, standard uncertainty \\
Student() & Student-1t distribution & Mean value, scale factor, degrees of freedom \\
Triangle() & Triangular distribution & Mean value, half-width \\
Trapez() & Cosine distribution & Mean value, half-width, beta factor \\
Ushape() & U-shaped (arcus-sinus) distribution & Mean value, half-width \\
Poisson() & Poisson distribution & Mean value \\
Ctrapez() & Iso-curvilinear trapezoidal distr. & Mean value, half-width, beta factor \\
Exponent() & Exponential distribution & Mean value \\
Gamma() & Gamma distribution & Mean value \\
Laplace() & Laplace distribution & Mean value, scale factor \\
\hline
\end{tabular}

The OMCE defines two quantity access functions to access the mean value and the standard uncertainty of named input quantities. The result of a quantity access function is a constant. These functions can be used together with the distribution functions to model dependencies between the parameters of different distributions.

\begin{tabular}{|c|l|l|}
\hline \multicolumn{3}{|c|}{ Predefined quantity access functions } \\
\hline Function & Description & Parameter \\
\hline val() & Value function to access the mean value of an input quantity & quantity name \\
$\mathrm{u}()$ & $\begin{array}{l}\text { Uncertainty function to access the standard uncertainty of } \\
\text { an input quantity }\end{array}$ & quantity name \\
\hline
\end{tabular}

OMCE supports evaluation models with multiple equations. The result of one equation may be used in the calculation of another equation. But no mathematical loops are allowed. It must be possible to evaluate the equation system in a linear execution order. The model will be checked for any mathematical loop before the simulation starts.

\subsubsection{Function minimum() and maximum()}

The functions minimum() $(\min ())$ and maximum() $(\max ())$ have one ore more parameter. The result of the function is the minimum or maximum of all the given values. These functions might produce a singular value in the PDF of the result if one of the parameter is a constant.

\subsubsection{Function pow ()}

The function pow () can be alternatively used instead of the exponential operator $* *$. The function has two parameters. The first is the basis and the second is the exponent.

\subsubsection{Function iff()}

The function iff() allows to switch between two values based on a specified condition. The function has three parameter. The first parameter is a logical expression. If the logical expression evaluates to True then the second parameter will be returned as a result. If the condition evaluates to False then the third parameter will be returned.

This function should be used with great care since it can produce serious discontinuities in the equation system. The use of this function might violate the basis for the application of GUM Supplement 1 [2] and the use is therefore not recommended. The function is provided for experimental purposes only.

\subsubsection{Logical expressions}

Logical expressions are used in constraints and as the first parameter of the iif()-function. They must evaluate to a boolean value (True or False). Usually logical expressions used in simulations compare 
data with limits or other data. The following comparison operators can be used:

\begin{tabular}{|l|c|c|c|}
\hline \multicolumn{3}{|c|}{ Comparison operators } \\
\hline Comparison & Operator & $\begin{array}{c}\text { Alternative } \\
\text { operator }\end{array}$ & Remarks \\
\hline equal to & $==$ &. eq & use with care in constraints \\
greater than & $\& g t ;$ &.$g t$ & \\
less than & $\& l t ;$ &.$l t$ & \\
greater than or equal to & $\& g t ;=$ & .ge & \\
less than or equal to & $\& l t ;=$ &.$l e$ & \\
not equal to & ! $=$ & .ne & \\
\hline
\end{tabular}

The characters < and > are used as special characters with XML-files and therefore cannot be entered directly in logical expressions. Use either the escape sequences \&gt; and \&lt; or the alternative dot operators instead.

Multiple comparisons can be combined with logical operators. The following operators are supported:

\begin{tabular}{|c|l|}
\hline \multicolumn{2}{|c|}{ Logical operators } \\
\hline Operator & Description \\
\hline and & logical and \\
or & logical or \\
not & logical complement \\
\hline
\end{tabular}

Some examples for valid logical expressions:

$$
\begin{aligned}
& X \text {.ge } 0 \\
& X_{-} 1 \text { \&lt; } X_{-} 2 \\
& (X . g e 1) \text { and }(X .1 t 2) \text { and } \operatorname{not}(Y . \text {. It } 0)
\end{aligned}
$$

The parenthesises are essential when combining comparisons.

\subsubsection{Symbol names}

Symbol names for quantities und equations are limited to the leters A...Z, a...z, the numbers $0 \ldots 9$ and the underscore character (" ."). Additionally, the @-character can be used, but it will be translated to a double underscore ("--") internally (e.g. A@A is the same as A_A). Therefore the @-character and the double underscore should not be used in the same model. The following words are reserved words and cannot be used as symbol names: and, as, assert, break, class, continue, def, del, elif, else, except, exec, finally, for, from, global, if, import, in, is, lambda, not, or, pass, print, raise, return, try, while, with, yield.

\subsection{Binary data file formats}

During the simulation a binary data file can be written (see option -wb). The OMCE supports two different binary formats.

\subsubsection{OMCE binary format}

The native OMCE binary format is a versatile binary format optimised for reading and writing speed. It has a block structure which corresponds to the processing block size of the data generation part of the simulation engine. The OMCE binary file has the following structure:

\section{OMCE binary file format}

\begin{tabular}{|l|l|l|}
\hline header & symbol table & binary data area \\
\hline $8 \times 4$ bytes & list of symbol names & runs $\times$ results $\times 8$ bytes \\
\hline
\end{tabular}


The header is 32 bytes long and contains $6 \times 32$-bit integer values and 8 unused bytes. The header has the following structure:

\begin{tabular}{|c|c|c|c|c|c|c|}
\hline \multicolumn{7}{|c|}{ OMCE binary header } \\
\hline-1330463557 & $m$ results & $n$ runs & block size $b$ & data offset & flags & not used \\
\hline 32-bit int. & 32-bit int. & 32-bit int. & 32-bit int. & 32 -bit int. & 32 bits & $2 \times 4$ bytes \\
\hline
\end{tabular}

The first integer in the header is a format identifier and contains the value -1330463557 . The following fields describe the data structure in the binary data area and the number of bytes used for other purposes (offset to the data). The offset to the data should be a multiple of 8 bytes.

If bit 0 in the flag word is one then a symbol table is included in the binary file. If a symbol table is present, it will directly follow the header. The symbol table contains ASCII character strings with names terminated by a zero character (00). The end of the list is an empty string or two zero characters. The symbol table is padded with additional zero characters to fill a block with a size of a multiple of 8 bytes.

The binary data contains a number of data blocks. A data block contains double precision floating point values (64-bit IEEE 754-1985) for one result only. The size is determined by the block size (see option -bs). If more than one result is specified the first data block of the second result will follow the first data block of the first result and so on until all first data blocks for all results are written. The same scheme will be followed up to the last block of the last result. In case that the total number of runs is not a multiple of the block size $b$, then the last block will be smaller than the standard block size.

\begin{tabular}{|c|c|c|c|c|}
\hline \multicolumn{5}{|c|}{ Binary data structure } \\
\hline data block 0, result 0 & $\ldots$ & data block 0 result $m$ & $\ldots$ & data block $k$ result $m$ \\
\hline$b \times 8$ bytes & $\ldots$ & $b \times 8$ bytes & $\ldots$ & $b_{k+1} \times 8$ bytes \\
\hline
\end{tabular}

A binary file reader needs to read the header first to find out about the data structure. The total number of floating point values (64-bit) in the binary file is the product of results and runs $m \times n$ given in the header. The reader can determine the number of full data blocks $k$ by dividing the number of runs by the block size given in the header $n / b$ (integer division). In case the total number of values is not a multiple of the block size, then the last block is smaller and has a block size $b_{k+1}$ which can be calculated from the number of runs modulo the block size $b_{k+1}=n \bmod b$ (modulo). After reading the header the reader should either read the symbol table if it is present or skip the number of bytes specified by the data offset in the header. The binary file contains information other than result values in this area. The value of the data offset might be zero. The unused integers in the header may be used later. The reader should not rely on them to have any specific value although they are set to zero by the OMCE so long they are not used.

A binary reader can distinguish between the OMCE binary format and the simple binary format (see next section)) by the value of the first entry in the header. The OMCE header contains a special negative number in this position while the number of runs $n$, the first integer in simple format, should always be positive.

\subsubsection{Simple binary format}

The simple binary format is an interleaving format like the OMCE format but with a fixed block size of 1 value per block. The simple binary file has the following structure:

\begin{tabular}{|c|c|c|c|c|c|c|}
\hline \multicolumn{7}{|c|}{ Simple binary file format } \\
\hline Header & result 0, value 0 & result 1, value 0 & $\ldots$ & result 0, value 1 & $\ldots$ & result $\mathrm{n}$, value $\mathrm{m}$ \\
$2 \times 4$ bytes & 8 bytes & 8 bytes & $\ldots$ & 8 bytes & $\ldots$ & 8 bytes \\
\hline
\end{tabular}

The header contains $2 \times 32$-bit integer values and is followed by a series of alternating values for all results. The header has the following structure:

\begin{tabular}{|c|c|}
\hline \multicolumn{2}{|c|}{ Simple binary header } \\
\hline$n$ runs & $m$ results \\
\hline 32-bit integer & 32 -bit integer \\
\hline
\end{tabular}


A binary file reader for the simple format may read the header first to find out about the number of runs and the number of results in the data file. While reading the data file the reader might separate the values for the different results and form larger blocks of binary values per result to optimize the later data handling. Reading and writing in simple format is less efficient than in OMCE format. The conversion from internal arrays in OMCE to the simple interleaving format of one value per block and back consumes significant computational effort.

\subsection{Text data file format}

OMCE supports the generation of two different output files in comma separated value format. The ohdfile contains the histogram data and the var-file contains the link between the binary data file and the result quantity names given in the model description in the omc-file or xml-file and optinal correlation information. An ohd-file is generated when $-\mathrm{a}=1$ is activated (default) and and a var-file is generated when -wv is set (deactivated by default). If a binary data file is written - wb $<>0$ then option - wv $=1$ should be set as well.

The content and the format of the text files (.var and .ohd) can be customized (see option -ofd and -vfd). The format of the different text output files is controlled by format definition files. The format definition files are text files themselves and they have a line based format. Every line in a format definition file is either a comment (starting with '\#'), an option (starting with '-') or a column definition (having an equal sign). All lines which are not options and do not contain an equal sign are ignored.

This is the format definition for the OMCE var-file format:

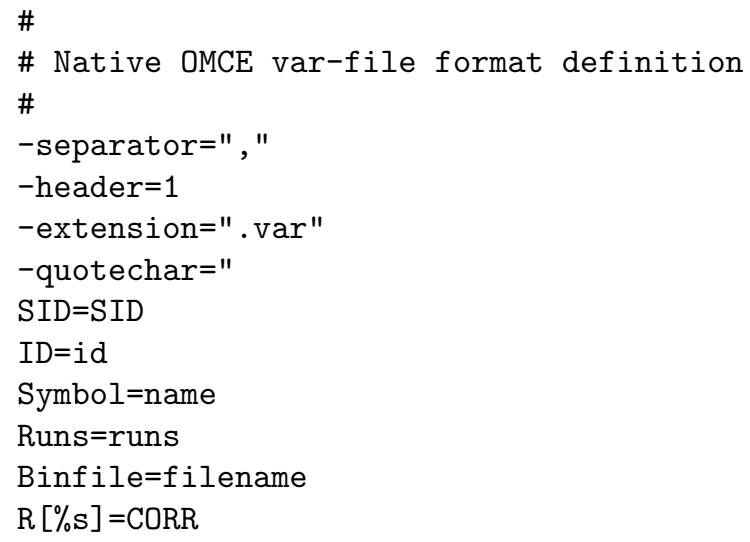

It defines a comma separated text file with the file name extension .var. The file has a header row and 5 or more columns named SID, ID, Symbol, Runs, Binfile and R [0] to R [n-1] for $n$ results.

The following options should be used in the format definition files:

-extension This option defines the extension to the output file name. The extension should start with a point on the line. The value should be put into quotes.

-header This option controls whether or not the file contains a header line with the column headings. A value not equal 0 will generate a header line.

-quotechar This option controls the quoting character. No quotes should be used with this option.

-separator This option controls the character to separate the columns in the data file. Use the combination $\backslash \mathrm{t}$ for the tab-character. The value should be put into quotes.

All lines which are neither a comment nor contain an option define a column in the data file if they contain an equal sign. The column name will be the string left of the equal sign and the content of the column is evaluated from the string right of the equal sign. The right side should contain a predefined symbol (see option - info $=3$ for a list of implemented symbols and the source code for details). 
The symbol BINS, CORR and IRCORR are used to specify the histogram data and correlation data. The string left of the equal sign must contain the sequence $\%$ s which will be replaced by an ident number or a name of an input quantity.

In case the string on the right side is quoted it will be taken literally and a fixed string is written in this column.

\section{$5 \quad$ Histogram viewer}

The OMCE includes a simple viewer to show the result in the text file containing the histogram. The viewer can be executed with the following command:

Viewer <filename.ohd> [<options $>$ ]

The extension of the histogram data file must be given. The viewer reads the histogram data file and shows histogram plots for the results as seperate plots. The plots can be saved in various graphic formats. To run the source code version of the viewer, the python module matplotlib [9] should be installed.

\section{$5.1 \quad$ Options}

The viewer supports the following options:

-mark The option -mark specifies vertical markers in the diagram to mark for example the expectation value and the coverage interval. The value of this option must be a list of marker specifications enclosed in square brackets [] A marker specification consists of a comma separated list of four values enclosed in parenthesis defining the column name, the color, the line style and the line width of the marker. The default value is:

$-\operatorname{mark}=[($ Mean, $\mathrm{b}$, dashed, 1$),($ Low95, $\mathrm{b}$, dashed, 1$),($ High95, $\mathrm{b}$, dashed, 1$)]$

The column names are referring to the names given in the ohd-file.

-max This option specifies the limit for the maximum number of plots that will be generated based on the result data file (.ohd). The default is $-\max =20$. If the number of results in the result data file exceeds the limit specified with this option, only the first results are plotted.

-sid This option specifies a range selection of simulation ident numbers (SID). The format of the option is - sid= $\langle\min \operatorname{sid}\rangle$ : $\langle\max \operatorname{sid}\rangle$ with $\langle\min \operatorname{sid}\rangle$ and $\langle\max$ sid $\rangle$ specify the lower and upper limit of simulation id's of the results which are plotted. The seperator is a colon (":"). If any of the values are obmitted then the minimum respectively the maximum SID in the file will be used. This option is only useful if the result data file contains data from different simulation runs.

\section{Adaptive mode}

The OMCE supports the automatic determination of the total number of Monte Carlo runs by the method described in the GUM Supplement 1 section 7.9 [2] (-am=0, default) or by alternate algorithms discuss in section 6.2. The adaptive mode is activated if the number of Monte Carlo blocks is set to zero (see option $-\mathrm{mcs}=0$ ).

\subsection{GUM Supplement 1 based algorithm}

The GUM S1 based algorithm performs the simulation in two steps. In the beginning a number of initial runs $n_{\mathrm{pr}}$, controlled by the option -pr, will be executed. After the initial runs the binning intervals are calculated and OMCE continues with performing additional runs. 
After every additional run the tolerances $T_{s}, T_{h}$ and $T_{l}$ are clculated. Based on these tolerances OMCE decides when to terminate the simulations. The tolerances are calculated based on the number of significant digits $n_{\mathrm{dig}}$ controlled by the option -sd and the tolerance divisor $d_{a}$ controlled by the option -ad.

For each data block $i$ the average $y_{i}$, the standard deviation $s_{i}$, and the interval limits $h_{i}$ and $l_{i}$ are calculated. Based on the $n_{\mathrm{r}}$ data blocks simulated so far, the tolerances are calculated by

$$
\begin{aligned}
T_{s} & =\frac{5 \times 10^{\operatorname{mag}(\bar{s})-n_{\mathrm{dig}}}}{d_{\mathrm{a}}} \\
T_{h} & =\frac{5 \times 10^{\operatorname{mag}(\bar{h}-\bar{y})-n_{\mathrm{dig}}}}{d_{\mathrm{a}}} \\
T_{l} & =\frac{5 \times 10^{\operatorname{mag}(\bar{y}-\bar{l})-n_{\mathrm{dig}}}}{d_{\mathrm{a}}}
\end{aligned}
$$

with

$$
\bar{s}=\sqrt{\frac{1}{n_{\mathrm{r}}} \sum_{i=1}^{n_{\mathrm{r}}} s_{i}^{2}}, \quad \bar{h}=\frac{1}{n_{\mathrm{r}}} \sum_{i=1}^{n_{\mathrm{r}}} h_{i}, \quad \bar{y}=\frac{1}{n_{\mathrm{r}}} \sum_{i=1}^{n_{\mathrm{r}}} y_{i}, \quad \bar{l}=\frac{1}{n_{\mathrm{r}}} \sum_{i=1}^{n_{\mathrm{r}}} l_{i} .
$$

The function $\operatorname{mag}(x)$ returns the order of magnitude of $x$.

During the additional simulation runs a multiple $k$ of the standard deviation of the mean of the three parameters $s_{i}, h_{i}$ and $l_{i}$ are compared with their tolerances. The simulation terminates if all parameters are within their limits. The multiplier $k$ is controlled by the option $-\mathrm{k}$. With the total number of simulation runs $n_{\mathrm{r}}$ the termination criteria are

$$
\begin{gathered}
b_{\text {MEAN }}=\left[\frac{k \times \operatorname{stddev}\left(y_{1} \ldots y_{n}\right)}{\sqrt{n}} \leq T_{s}\right] \\
b_{\text {UNCERTAINTY }}=\left[\frac{k \times \operatorname{stddev}\left(s_{1} \ldots s_{n}\right)}{\sqrt{n}} \leq T_{s}\right] \\
b_{\text {LIMITS }}=\left[\frac{k \times \operatorname{stddev}\left(h_{1} \ldots h_{n}\right)}{\sqrt{n}} \leq T_{h}\right] \wedge\left[\frac{k \times \operatorname{stddev}\left(l_{1} \ldots l_{n}\right)}{\sqrt{n}} \leq T_{l}\right] .
\end{gathered}
$$

The function $\operatorname{stddev}\left(x_{1}, \ldots, x_{n}\right)$ returns the experimental standard deviation of the valid data in the series $x_{1} \ldots x_{n}$ (see Section 10.1).

Which criterion is actually in use is controlled by the option -at. The value of this option is a bit combination which enables the different criteria.

$$
b_{\text {TERMINATION }}=\left(\neg \operatorname{bit}(0) \vee b_{\text {MEAN }}\right) \wedge\left(\neg \operatorname{bit}(1) \vee b_{\text {UNCERTAINTY }}\right) \wedge\left(\neg \operatorname{bit}(2) \vee b_{\text {LIMITS }}\right) .
$$

The function bit() returns a logical value true if the bit in option -at with the number given as an argument is set and the value false otherwise. By default all termination criteria are used $(-$ at $=7)$.

\subsection{Alternate algorithms}

The OMCE supports alternate adaptive algorithms for the determination of the total number of Monte Carlo runs. The algorithms are chosen by the option -am. The standard GUM S1 algorithm is the default $(-\mathrm{am}=0)$.

The numerical accuracy of all algorithms is controlled by the same options. The number of significant digits $n_{\text {dig }}$ are controlled by the option -sd and the tolerance divisor $d_{a}$ is controlled by the option -ad. The probability level $p$ for the test is controlled by the option - af and the block size $n_{b}$ is controlled by the option -bs. 
The algorithms selected with options $-\mathrm{am}=1$ and $-\mathrm{am}=2$ use a modified Stein-method [5]. After the initial $n_{\text {pr }}$ simulation blocks controlled by option $-\mathrm{pr}$, this methods estimates the total number of runs bases on a confidence interval.

The algorithms selected with options $-\mathrm{am}=3$ and $-\mathrm{am}=4$ are based on the fact that the numerical accuracy is in principle defined relative to the variance, which allows the exact knowledge about the number of runs needed for normally distributed results.

The accuracy definition in GUM S1 is defined as half of the least significant digit and therefore the relative accuracy is dependent on the actual digits of the uncertainty (between $0.5 \%$ for the digits "99" and $5 \%$ for digits "10") the number of runs needed to meet this specification is dependent on the digits of the uncertainty.

The algorithms selected with options $-a m=1$ and $-a m=3$ use the definition of the numerical accuracy as defined in GUM S1 while the algorithm selected with option -am=2 and -am=4 use always the highest accuracy (assuming the digits of the uncertainty are always "99").

The algorithm selected with option $-a m=3$ uses a simple scheme to adapt the number of runs according to the digits of the uncertainty.

At the end all algorithms perform a statistical test to ensure that the result meets the numerical accuracy even in cases when the results are not normally distributed.

The alternate algorithms support also the option -at to limit the checking of the statistical parameter to the mean (bit 0) or the variance (bit 1). Bit 2 has no function with these algorithms.

\subsubsection{Sub-blocking}

A sub-block mechanism is used to improve the statistical properties of the statistical evaluations. The result data blocks of size $n_{\mathrm{b}}$ are divided in sub-blocks of size $n_{\mathrm{sb}}$ (controlled by option -sbs) resulting in $n_{\mathrm{s}}=n_{\mathrm{r}} \times n_{\mathrm{b}} / n_{\mathrm{sb}}$ sub-blocks for $n_{\mathrm{r}}$ simulated blocks. The standard deviation of the values in sub-block number $i$ is $s_{i}\left(y_{\mathrm{sb}}\right)$ and the mean value of the same block is $\bar{y}_{\mathrm{sb}}(i)$. The degrees of freedom are improved by the ratio $n_{\mathrm{b}} / n_{\mathrm{sb}}$. The sub-blocking is implemented efficiently without any data copying by creating a second view on the data. The sub-blocking is advantageous compare to a reduction of the block size because the performance of a Python based simulator and the accuracy of the evaluation of the coverage intervals are dependent on a large block size $(\geq 10000)$.

\subsubsection{Establishing the statistical confidence level}

As proposed in [5] OMCE uses a $t$-factor, based on the number of sub-blocks $n_{\mathrm{s}}$, to achieve the numerical accuracy. As long as the sub-block mean values $\bar{y}_{\mathrm{sb}}(i)$ and the sub-block standard deviations $s_{i}\left(y_{\mathrm{sb}}\right)$ can be considered as random samples of a distribution with an existing variance, the variance of the overall mean and the overall variance will be improved by a factor of $n_{\mathrm{s}}$. Therefore it is possible to use the function $\mathrm{N}_{\mathrm{sb}}(s, d)$ to calculate an estimate of the number of blocks needed to achieve the numerical accuracy based on the standard deviation $s$ and the tolerance $d$.

$$
\mathrm{N}_{\mathrm{sb}}(s, d)=\max \left[\operatorname{int}\left(\frac{n_{\mathrm{sb}}}{n_{\mathrm{b}}} \times \frac{s^{2} \times t_{p}\left(n_{\mathrm{s}}-1\right)^{2}}{d^{2}}+0.5\right), 1\right]
$$

The function $\max \left[n_{1}, \ldots, n_{l}\right]$ returns the maximum value of the values $n_{1} \ldots n_{l}$ and the function $t_{p}\left(n_{\mathrm{s}}-1\right)$ returns the $t$-factor based on the probability $p$ and the degrees of freedom $n_{\mathrm{s}}-1$. In case $n_{\mathrm{s}}$ is large $(>100)$, the $t$-factor could be replaced by quantile function of a normal distribution.

\subsubsection{Numerical tolerance}

The numerical tolerances can be either established by using the GUM S1 definition that the tolerance is half of the least significant digit of the uncertainty or the tolerance can be defined as relative to the uncertainty of the result. 
The tolerance for the mean value $d_{\mathrm{m}}$ based on the GUM S1 definition is

$$
d_{\mathrm{m}}=0.5 \times \frac{10^{\operatorname{mag}(u(y))+1} \times 10^{-n_{\mathrm{dig}}}}{d_{a}}
$$

and the relative tolerance is dependent on the digits of the uncertainty (between $0.5 \%$ for the digits "99" and $5 \%$ for digits "10"). The function $\operatorname{mag}(x)$ returns the order of magnitude of $x$. This tolerance is used by the the algorithms selected with options -am=1 and -am=3.

For the algorithms selected with options $-a m=2$ and $-a m=4$, the relative tolerance is fixed (assuming the digits of the uncertainty are always "99").

$$
d_{\mathrm{m}}=0.5 \times \frac{u(y) \times 10^{-n_{\mathrm{dig}}}}{d_{a}} .
$$

The tolerance for the variance $d_{\mathrm{v}}$ is for all algorithms

$$
d_{\mathrm{v}}=2 \times u(y) \times d_{\mathrm{m}} .
$$

\subsubsection{Predicting the number of simulation blocks}

The algorithms selected with options $-\mathrm{am}=3$ and $-\mathrm{am}=4$ are based on the fact that the relative numerical accuracy of the mean value is in principle predictable if the mean value is calculated based on random samples from a distribution which has an existing variance.

Based on the number of digits $n_{\text {dig }}$ and the tolerance divisor $d_{a}$ the relative tolerance factor $m$ can be calculated.

$$
m=10^{n_{\mathrm{dig}}} \times d_{a}
$$

Assuming that the number of simulations is large $(\gg 10000)$ we can assume that the mean value is normally distributed and we can use the quantiles of a normal distribution to calculate a confidence interval. The variance of the mean value is proportional to $1 / n$. Therefore we can predict the number of simulation runs needed to establish the relative accuracy $m$. This number divided by the block size $n_{\mathrm{b}}$ and rounded to the nearest integer greater than or equal to one is the number of simulation blocks $n_{0}$ to achieve the relative accuracy $1 / m$.

$$
n_{0}=\max \left[\operatorname{int}\left(\frac{m^{2} \times \operatorname{norm}(1-(1-p) / 2)^{2}+n_{\mathrm{b}}-1}{n_{\mathrm{b}}}\right), 1\right]
$$

The function norm $(p)$ calculates the quantile of the probability $p$ of a normal distribution and the function int $(x)$ returns the integer part of $x$.

The algorithm selected with option -am=3 uses a multiple of $n_{0}$ to adapt the number of runs to the digits of the uncertainty.

The relative accuracy $m$ is the correct numerical accuracy for the uncertainty digits " 50 " based on the GUM S1 definition. The correct numerical accuracy for the uncertainty digits "99" which is needed for the algorithm selected with option $-\mathrm{am}=4$, results in a relative accuracy of $2 \times m$ and therefore in a number of simulation blocks $n_{1}$.

$$
n_{1}=\max \left[\operatorname{int}\left(\frac{4 \times m^{2} \times \operatorname{norm}(1-(1-p) / 2)^{2}+n_{\mathrm{b}}-1}{n_{\mathrm{b}}}\right), 1\right]
$$

The value of $n_{0}$ is the number of simulation blocks needed to improve the variance of the mean value by $m$ and $n_{1}$ is the number of simulation blocks needed to improve the variance of the mean value by $2 \times m$. 


\subsubsection{Algorithms based on the Stein-method}

The algorithms selected with options $-\mathrm{am}=1$ and $-\mathrm{am}=2$ use a modified Stein-methods [5]. After the initial $n_{\text {pr }}$ simulation blocks controlled by option -pr, this methods estimate the total number of runs bases on confidence intervals for the mean and the variance using the function $\mathrm{N}_{\mathrm{sb}}$ defined in Section 6.2.2.

$$
n_{\mathrm{r}}=\max \left[\mathrm{N}_{\mathrm{sb}}\left(\operatorname{stddev}\left(\bar{y}_{\mathrm{sb}}(1), \ldots, \bar{y}_{\mathrm{sb}}\left(n_{\mathrm{s}}\right)\right), d_{\mathrm{m}}\right), \mathrm{N}_{\mathrm{sb}}\left(\operatorname{stddev}\left(s_{1}^{2}\left(y_{\mathrm{sb}}\right), \ldots, s_{n_{\mathrm{s}}}^{2}\left(y_{\mathrm{sb}}\right)\right), d_{\mathrm{v}}\right)\right] .
$$

The methods execute $n_{\mathrm{r}}-n_{\mathrm{pr}}$ additional runs and perform at the end the statistical test discussed in Section 6.2.7.

\subsubsection{Algorithms based on a prediction of the needed runs}

The algorithms selected with options $-\mathrm{am}=3$ and $-\mathrm{am}=4$ are based on a prediction of the runs needed (see Section 6.2.4).

If option $-\mathrm{am}=3$ or $-\mathrm{am}=4$ are selected then option $-\mathrm{pr}$ will be ignored and a number of $n_{0} \times n_{b}$ simulations are run as initial runs.

After the initial $n_{0}$ runs the algorithm selected with option -am=4 executes a fixed number of $n_{1}-n_{0}$ runs as additional runs.

The algorithm selected with option -am=3 uses the significant digits $d_{u}$ of the uncertainty of the result $u(y)$ determined by the simulations so far to evaluate the need for additional runs.

$$
d_{u}=\frac{u(y)}{10^{\operatorname{mag}(u(y))-1}}
$$

The function mag $(x)$ returns the order of magnitude of $x$.

The value of $d_{u}$ is used to judge if additional runs are needed.

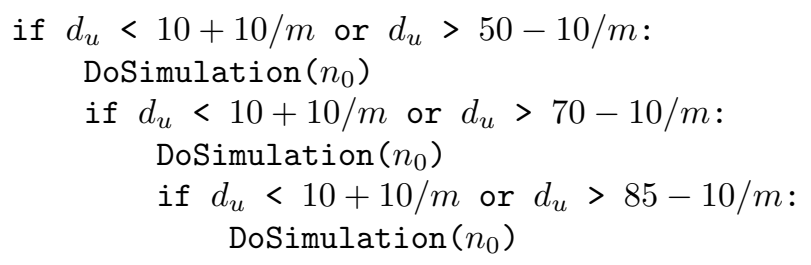

The function DoSimulation $(n)$ executes $n$ simulation blocks and $m$ is the relative tolerance factor defined in Section 6.2.4. Hint: The value of $d_{u}$ is re-evaluated after the execution of DoSimulation().

After the execution of the initial runs and the additional runs in total $n_{\mathrm{r}}$ simulation blocks have been executed. At the end both algorithms perform the statistical test (see next section).

\subsubsection{Statistical end test}

The improved adaptive algorithms selected by the option -am $>0$ test the simulation results at the end to verify that the standard deviation of the mean and the standard deviation of the variance meet the tolerance specified by $n_{\mathrm{dig}}, d_{a}$ and $p$. The sub-blocking mechanism (controlled by option -sbs) is used to improve the statistical properties of this test (see Section 6.2.1).

The test verifies that the number of runs is greater than or equal to the square of the confidence interval based on a $t$-factor $t_{p}\left(n_{\mathrm{s}}-1\right)$ and the standard deviation divided by the square of the calculated tolerance.

The test uses the function $\mathrm{N}_{\mathrm{sb}}(s, d)$ defined in Section 6.2.2 with the tolerances $d_{\mathrm{m}}$ and $d_{\mathrm{v}}$ discussed in Section 6.2.3.

The minimum number of runs $n_{\min }$ ensuring that the statistical properties for the mean value and the variance are met is

$$
n_{\text {min }}=\max \left[\mathrm{N}_{\mathrm{sb}}\left(\operatorname{stddev}\left(\bar{y}_{\mathrm{sb}}(1), \ldots, \bar{y}_{\mathrm{sb}}\left(n_{\mathrm{s}}\right)\right), d_{\mathrm{m}}\right), \mathrm{N}_{\mathrm{sb}}\left(\operatorname{stddev}\left(s_{1}^{2}\left(y_{\mathrm{sb}}\right), \ldots, s_{n_{s}}^{2}\left(y_{\mathrm{sb}}\right)\right), d_{\mathrm{v}}\right)\right] .
$$


The function $\operatorname{stddev}\left(x_{1}, \ldots, x_{n}\right)$ returns the experimental standard deviation of the valid data in the series $x_{1} \ldots x_{n}$ (see Section 10.1) and the function $\max \left[n_{1}, \ldots, n_{l}\right]$ returns the maximum value of the values $n_{1} \ldots n_{l}$.

WAn iterative test is used to verify the statistical properties since the number of sub-blocks $n_{s}$ is large $(>100)$.

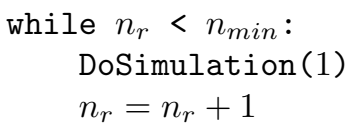

Hint: The value of $n_{\min }$ is re-evaluated after every execution of DoSimulation().

In case the sub-block size is large enough $\left(n_{\mathrm{sb}} \geq 200\right)$ and the distribution of the result $y$ is not extreme and the variance of the variance exists, one can assume a close to normal distribution for the mean values $\bar{y}_{\mathrm{sb}}(i)$ and the variances $s_{i}^{2}\left(y_{\mathrm{sb}}\right)$ of the sub-blocks (Central Limit Theorem). In this case the standard deviations of the averages will be reduced by a factor of $n_{\mathrm{s}}$ and the total number of runs can be estimated or predicted by the algorithms very precisely. Then the statistical test at the end will find only in a small number of cases that the number of runs $n_{r}$ is too small $(<5 \%$, one additional block). The need for a larger number of runs during the end test might be an indication that the Central Limit Theorem is not applicable for the specific simulation problem.

\section{$7 \quad$ Running multiple simulations}

The OMCE is a command line program that is designed to be used in batch mode. In case it is necessary to execute a Monte Carlo simulation several times based on the same underlying model, one can either use the batch processing of the operating system and call OMCE several times or one can use the section <Simulations > in the omc-file to define multiple simulations. If some of the parameters need to be changed between the runs then they must be defined in the section <Parameters>. Using the batch processing of the operating system has the advantage that several simulations can run in parallel and that the same batch processing could be used for different models by changing only the omc-file name. Using the section <Simulations> has the advantage to combine everything in one input file and to generate one output file.

The following example demonstrates the use of the section Simulations to repeat the simulation three times and re-assigning the value of $\mathrm{P}$ to $1.0,4.0$ and 9.0 .

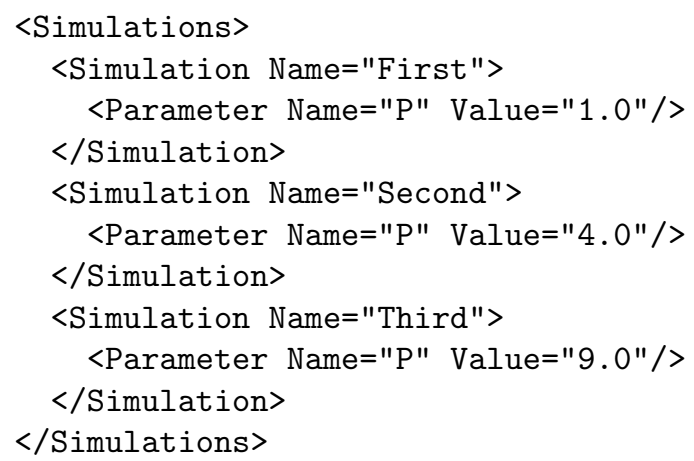

Alternatively, the same result can be achieved by using the element Loop.

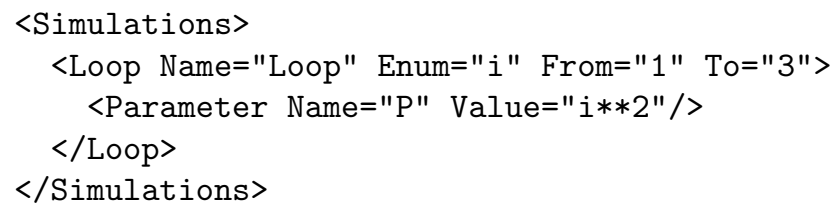


The value of a parameter can be evaluated using mathematical operators and predefined mathematical functions.

It should be noted that the parameter re-assignment in the section simulations override the parameter values given in the command line. So care should be taken if a omc-file with a section simulations is used together with batch processing.

\section{Input correlation coefficients}

The matrix based on the correlations coefficients given in the omc-file should be positive semi-definite. This is checked by Cholesky decomposition and eigenvalue decomposition. In cases when the matrix found to be not positive semi-definite the matrix is corrected by the chosen correction procedure $(-\mathrm{mcp})$. The default procedure is to shift all eigenvalues which are smaller a given limit $(-\mathrm{sev})$ to the limit and compose a new correlation matrix. A warning message together with the values of the modified correlation matrix is printed if a modification is necessary. The modification of the matrix is controlled by applying a norm [10] to the difference of the shifted and the non-shifted matrix. The norm must be smaller than a given limit (see option -e2) or the engine terminates with an error message.

\section{$9 \quad$ Analysis of correlation}

The OMCE can analyse the correlation in the results data. The analysis is controlled by the option -ac. The correlation is evaluated pair-wise by

$$
r\left(q_{i}, q_{j}\right)=\frac{1}{(n-1) s\left(q_{i}\right) s\left(q_{j}\right)} \sum_{l=1}^{n}\left(q_{i, l}-q_{i}\right)\left(q_{j, l}-q_{j}\right)
$$

with $q_{i, l}$ and $q_{j, l}$ being the data of the two results with the average $q_{i}$ and $q_{j}$ and the standard deviation $s\left(q_{i}\right)$ and $s\left(q_{j}\right)$. The correlation is evaluated for every block and the result correlation matrix is the average over all blocks. For $m$ results $\left(m^{2}-m\right) / 2$ correlation coefficients are calculated. The correlation can be printed with the option $-1 \mathrm{c}=2$ or the data can be written to the output files.

\section{Constraints on the domain of the functions}

The evaluation models given in the section <Equations> might be constrained in their domain. The use of the function sqrt() for example forms such constraint since the argument of the function cannot be negative. Any values violating any domain of the functions involved will be marked as not valid and the values will be ignore in further calculations. In addition, constraints specified in the section <Constraints $>$ might restrict the domain and invalidate some values as well. As a consequence the number of valid values per block might be smaller than the number of trails. The number of runs in the statistical data file is always the number of valid values used to evaluate the result.

Since the statistical treatment is relying on a sufficiently large number of values per block, the ratio between valid values and trails is monitored. The simulation will be aborted as soon as the ratio drops below the limit defined by $-\mathrm{nl}$ (default $50 \%$ ). It is possible to reduce this limit, but it is advisable to increase the block-size (-bs) at the same time to guarantee that statistical treatment has still a good performance.

The correlation analysis can only be performed based on valid common data. Any invalid values in one quantity will automatically discard the values for al quantities from the data set. The ratio between the total number of values and the number of valid values is monitored. The simulation will be aborted as soon as the ratio drops below the limit defined by - nc (default $50 \%$ ).

The analysis of correlation can be disabled with the option $-\mathrm{ac}=0$ if the correlation information is not needed. It is also possible to reduce the limit with option -nc and increase the block-size sufficiently. 
Non-valid data will reduce the performance of the simulation and therefore should be avoided if possible. The result will only be calculated from the valid data and all data marked as non-valid will not contribute to the result and therefore should not be calculated at all.

\subsection{Mean value and uncertainty calculation}

In case some of the data in a result data block $i$ become not valid because of constraints, the effective block size of block $i$ is reduced $\left(n_{\mathrm{eff}}(i) \leq n_{\mathrm{b}}\right)$.

The total number of simulation results $n$ in $n_{\mathrm{r}}$ simulation blocks is

$$
n=\sum_{i=1}^{n_{\mathrm{r}}} n_{\mathrm{eff}}(i)
$$

and the average effective blocksize $\bar{n}_{\mathrm{eff}}$ is

$$
\bar{n}_{\mathrm{eff}}=\frac{1}{n_{\mathrm{r}}} \sum_{i=1}^{n_{\mathrm{r}}} n_{\mathrm{eff}}(i)
$$

If $y[i]$ is the set of valid results for result data block $i$ then the effective mean value $\bar{y}_{\text {eff }}(i)$ of this set is

$$
\bar{y}_{\mathrm{eff}}(i)=\frac{1}{n_{\mathrm{eff}}(i)} \sum_{j=1}^{n_{\mathrm{eff}}(i)} y[i]_{j}
$$

and the effective variance $s_{\text {eff }}^{2}(i)$ of the data in this set is

$$
s_{\mathrm{eff}}^{2}(i)=\frac{1}{n_{\mathrm{eff}}(i)-1} \sum_{j=1}^{n_{\mathrm{eff}}(i)}\left(y[i]_{j}-\bar{y}_{\mathrm{eff}}(i)\right)^{2} .
$$

The overall mean value of the result $\bar{y}$ is

$$
\bar{y}=\frac{1}{n} \sum_{i=1}^{n_{\mathrm{r}}} n_{\mathrm{eff}}(i) \times \bar{y}_{\mathrm{eff}}(i)
$$

and the variance $u^{2}(y)$ is

$$
u^{2}(y)=\frac{1}{n-1} \sum_{i=1}^{n_{\mathrm{r}}} \sum_{j=1}^{n_{\mathrm{eff}}(i)}\left(y[i]_{j}-\bar{y}\right)^{2}
$$

which is equivalent of calculating

$$
u^{2}(y)=\frac{1}{n-1}\left[\sum_{i=1}^{n_{\mathrm{r}}}\left(n_{\mathrm{eff}}(i)-1\right) \times s_{\mathrm{eff}}^{2}(i)+\sum_{i=1}^{n_{\mathrm{r}}} n_{\mathrm{eff}}(i) \times\left(\bar{y}_{\mathrm{eff}}(i)-\bar{y}\right)^{2}\right] .
$$

The average of the effective variance $\bar{s}_{\text {eff }}^{2}$ is

$$
\bar{s}_{\mathrm{eff}}^{2}=\frac{1}{n} \sum_{i=1}^{n_{\mathrm{r}}} n_{\mathrm{eff}}(i) \times s_{\mathrm{eff}}^{2}(i) .
$$

The variance $s^{2}\left(\bar{y}_{\text {eff }}()\right)$ of the block mean values $\bar{y}_{\text {eff }}(i)$ for $i=1 \ldots n_{\mathrm{r}}$ is

$$
s^{2}\left(\bar{y}_{\mathrm{eff}}()\right)=\frac{1}{\bar{n}_{\mathrm{eff}} \times\left(n_{\mathrm{r}}-1\right)} \sum_{i=1}^{n_{\mathrm{r}}} n_{\mathrm{eff}}(i) \times\left(\bar{y}_{\mathrm{eff}}(i)-\bar{y}\right)^{2}
$$

and the variance of the effective variances of the sets is

$$
s^{2}\left(s_{\mathrm{eff}}^{2}\right)=\frac{1}{\bar{n}_{\mathrm{eff}} \times\left(n_{\mathrm{r}}-1\right)} \sum_{i=1}^{n_{\mathrm{r}}} n_{\mathrm{eff}}(i) \times\left(s_{\mathrm{eff}}(i)-\bar{s}_{\mathrm{eff}}\right)^{2} .
$$


The nominal variance $s_{\text {nom }}^{2}\left(\bar{y}_{\text {eff }}()\right)$ of the block mean values $\bar{y}_{\text {eff }}(i)$ for $i=1 \ldots n_{\mathrm{r}}$ is

$$
s_{\text {nom }}^{2}\left(\bar{y}_{\mathrm{eff}}()\right)=\frac{\bar{n}_{\mathrm{eff}}}{n_{\mathrm{b}}} \times s^{2}\left(\bar{y}_{\mathrm{eff}}()\right)
$$

The nominal variance $s_{\text {nom }}^{2}(y())$ is an estimate of the variance one would have got in case all blocks would have had the nominal size $n_{\mathrm{b}}$.

\section{Testing and validation}

OMCE makes use of the numerical library Numpy whenever possible. So the quality of the numerical calculations are based on this library. The library is widely used and tested and is considered reliable in the context of OMCE testing and validation.

The examples given in GUM Supplement 1 are included as examples and the results can be compared with the published results.

Output (.ohd) files for all examples can be found in OMCE\examples $\backslash r$ ef $\backslash$. They were generated with a seeded random generator $(-$ seed $=1)$. This is used as a basic regression test during the development and it should be used to verify the installation. The windows batch file !run_all_OMCE.bat runs all examples and compares the result with the reference results.

\section{Performance}

The OMCE is optimized for an efficient calculation of models with large equation systems. During the simulation a major part of the computational power of the system will be used. The memory consumption is dependent on the model and is about $1 \mathrm{MB}$ per input quantity and result.

The binary data file can be rather large without being of much use after the simulation. By default the data file will be not created. Although the binary data file is written in parallel with the calculation, the time for writing the binary data file might contribute significantly to the overall execution time if the data calculation part is significantly faster than the data writing part. At the end the OMCE has to wait until all data blocks are written to the binary file before the simulation can be completed.

\subsection{Random number generation}

The OMCE uses the Mersenne Twister algorithm from the NumPy package. The Mersenne Twister algorithm has a large period $\left(2^{19937}-1\right)$, creates random floats to full 53-bit precision, and has been widely tested.

\section{References}

[1] Guide to the Expression of Uncertainty in Measurement, International Organization for Standardization, 1995

[2] Expression of Uncertainty in Measurement Supplement 1: Numerical Methods for the Propagation of Distributions, JCGM Working Group of the Expression of Uncertainty in Measurement, 2006

[3] Stackless Python - extended Python for thread-based programming, open source software package, http://www.stackless.com

[4] NumPy - Numerical Python extension, open source software package, http://numpy.scipy.org

[5] G. Wübbeler, P. M. Harris, M. G. Cox, C. Elster: A two-stage procedure for determining the number of trials in the application of a Monte Carlo method for uncertainty evaluation, Metrologia 47(3): 317-324, 2010, doi:10.1088/0026-1394/47/3/023 
[6] N. J. Higham: Computing the nearest correlation matrix - A problem from finance. IMA J. Numer. Anal., 22(3):329-343, 2002

[7] R. J. Hyndman, Y. Fan: Sample Quantiles in Statistical Packages, The American Statistician Vol. 50, No. 4 (1996), pp. 361-365, http://www.jstor.org/stable/2684934

[8] R. N. Kacker, J. F. Lawrence: Rectangular distribution whose width is not exactly known: isocurvilinear trapezoidal distribution, Metrologia 46: 254260, 2009, doi:10.1088/0026-1394/46/3/012

[9] matplotlib - 2D plotting library, open source software package, http://matplotlib.sourceforge.net/

[10] S. K. Mishra: Optimal solution of the nearest correlation matrix problem by minimization of the maximum norm, 2004, http://mpra.ub.uni-muenchen.de/1783/ 


\section{A Program exit codes}

\begin{tabular}{|c|c|}
\hline \multicolumn{2}{|r|}{ Program exit codes } \\
\hline Exit Code & Error message \\
\hline 001 & Error: Option <opt> is not valid! \\
\hline 002 & Error: File not found $(<$ filename $>)$ ! \\
\hline 003 & Error: It is not a file (<string $\rangle)$ ! \\
\hline 004 & Error: The histogram bins must increase monotonically.! \\
\hline 005 & Error: Option <option> should be a number (<value>)! \\
\hline 006 & Error: Option out of range $(<$ option $\rangle=\langle$ from $\rangle \ldots<$ to $\rangle)$ ! \\
\hline 007 & Error: The attribute mcsimulations is not set correctly (<value $\rangle)$ ! \\
\hline 008 & Error: The binary data format <number > is not supported! \\
\hline 009 & Error: Format $<$ format $>$ is not supported! \\
\hline 010 & Error: Mathematical loop in process list! \\
\hline 011 & Error: Input quantity <name> is not defined! \\
\hline 012 & Error: Duplicated quantity name (<name>)! \\
\hline 013 & Error: Error in expression: <expression>! \\
\hline 014 & Error: Quantity <name> in Result <name> is unknown! \\
\hline 015 & Error: Model Parameter <parameter > not supported! \\
\hline 016 & Error: Correlation matrix is not positive semi-definite! \\
\hline 017 & Error: Quantity name unknown in correlation entry (<name>)! \\
\hline 018 & Error: Correlations are not supported for non-Gaussian Quantities (<name>)! \\
\hline 019 & Error: Correlation coefficient out of range $\mathrm{r}(\langle\mathrm{name}\rangle,\langle$ name $\rangle)=\langle$ value $\rangle$ ! \\
\hline 020 & Error: Matrix modification exceeds limit (-e2=<limit $>)$ ! \\
\hline 021 & $\begin{array}{l}\text { Error: Diagonal elements of the correlation matrix must be one } \\
{[\mathrm{r}(\langle\text { name }\rangle,\langle\text { name }\rangle)=\langle\text { value }>]}\end{array}$ \\
\hline 022 & Error: Quantity name <name> is a reserved symbol! \\
\hline 023 & Error: Quantity <name>, parameter <param>, syntax error in <value $\rangle$ ! \\
\hline 024 & Error: Parameter of function $<$ name $>()$ is not a constant! \\
\hline 025 & Error: Function <name> is used without parameter! \\
\hline 026 & Error: Quantity unknown for val(<name>)! \\
\hline 027 & Error: The uncertainty for a t-distribution with $\operatorname{dof}<3$ is not defined in $\mathrm{u}(<$ name $>)$ ! \\
\hline 028 & Error: Quantity unknown for $\mathrm{u}(<$ name $>)$ ! \\
\hline 029 & Error: Grammar type not supported (<type-id>)! \\
\hline 030 & Error: Function $<$ name $>()$ is used recursively in its definition! \\
\hline 031 & Error: Quantity $<$ name $>$ is defined as parameter and as global in function $<$ name $>()$ ! \\
\hline 032 & Error: Global quantity $<$ name $>$ in function $<$ name $>()$ is not defined! \\
\hline 033 & Error: Quantity <name> in function <name $>()$ is not defined as parameter or global! \\
\hline 034 & Error: Unknown command line parameter name <name>! \\
\hline 035 & Error: Quantity <name>, parameter <param>, value <value> is not an integer! \\
\hline 036 & Error: Quantity <name>, parameter <param>, parameter unknown in 〈value $\rangle$ ! \\
\hline 037 & $\begin{array}{l}\text { Error: Quantity <name>, parameter <param>, \%-character is not supported } \\
(<\text { value }>) \text { ! }\end{array}$ \\
\hline 038 & Error: Quantity <name>, parameter <param>, syntax error in <value>! \\
\hline 039 & Error: Quantity name <name> is a predefined function! \\
\hline 040 & Error: Quantity name <name> is a predefined symbol! \\
\hline 041 & Error: Quantity name <name> is a reserved name! \\
\hline 042 & Error: Quantity name <name> is the name of a user defined function! \\
\hline 043 & Error: Parameter name <name $>$ is a predefined function! \\
\hline 044 & Error: Parameter name <name> is a reserved name! \\
\hline 045 & $\begin{array}{l}\text { Error: Quantity <name }>\text { in }\langle\text { Discrete }>\text { or }<\text { Import }>\text { element is unknown in the } \\
\text { bin-file }\langle\text { filename }>\text { ! }\end{array}$ \\
\hline 046 & Error: Index unknown! \\
\hline 047 & Error: Result name <name $>$ is used twice in result section! \\
\hline
\end{tabular}




\begin{tabular}{|c|c|}
\hline \multicolumn{2}{|r|}{ Program exit codes } \\
\hline Exit Code & Error message \\
\hline 001 & Error: Option <opt> is not valid! \\
\hline 002 & Error: File not found ( $\langle$ filename $\rangle)$ ! \\
\hline 003 & Error: It is not a file (<string $>)$ ! \\
\hline 004 & Error: The histogram bins must increase monotonically!! \\
\hline 005 & Error: Option <option> should be a number (<value>)! \\
\hline 006 & Error: Option out of range $(\langle$ option $\rangle=\langle$ from $\rangle \ldots\langle$ to $\rangle) !$ \\
\hline 007 & Error: The attribute mcsimulations is not set correctly (<value $\rangle)$ ! \\
\hline 008 & Error: The binary data format <number > is not supported! \\
\hline 009 & Error: Format $<$ format $>$ is not supported! \\
\hline 010 & Error: Mathematical loop in process list! \\
\hline 011 & Error: Input quantity <name> is not defined! \\
\hline 012 & Error: Duplicated quantity name (<name>)! \\
\hline 013 & Error: Error in expression: <expression>! \\
\hline 014 & Error: Quantity <name> in Result <name> is unknown! \\
\hline 015 & Error: Model Parameter <parameter> not supported! \\
\hline 016 & Error: Correlation matrix is not positive semi-definite! \\
\hline 017 & Error: Quantity name unknown in correlation entry (<name $>)$ ! \\
\hline 018 & Error: Correlations are not supported for non-Gaussian Quantities (<name>)! \\
\hline 019 & Error: Correlation coefficient out of range $\mathrm{r}(\langle$ name $\rangle,\langle$ name $\rangle)=\langle$ value $\rangle$ ! \\
\hline 020 & Error: Matrix modification exceeds limit (-e $2=<$ limit $>)$ ! \\
\hline 021 & $\begin{array}{l}\text { Error: Diagonal elements of the correlation matrix must be one } \\
{[\mathrm{r}(\langle\mathrm{name}\rangle,\langle\text { name }\rangle)=\langle\text { value }\rangle]}\end{array}$ \\
\hline 022 & Error: Quantity name <name> is a reserved symbol! \\
\hline 023 & Error: Quantity <name>, parameter 〈param>, syntax error in 〈value $\rangle$ ! \\
\hline 024 & Error: Parameter of function $<$ name $>()$ is not a constant! \\
\hline 025 & Error: Function <name> is used without parameter! \\
\hline 026 & Error: Quantity unknown for val(<name>)! \\
\hline 027 & Error: The uncertainty for a t-distribution with $\operatorname{dof}<3$ is not defined in $\mathrm{u}(<$ name $>)$ ! \\
\hline 028 & Error: Quantity unknown for $\mathrm{u}(<$ name $>)$ ! \\
\hline 029 & Error: Grammar type not supported (<type-id>)! \\
\hline 030 & Error: Function $<$ name $>()$ is used recursively in its definition! \\
\hline 031 & Error: Quantity $<$ name $>$ is defined as parameter and as global in function $<$ name $>()$ ! \\
\hline 032 & Error: Global quantity <name $>$ in function $<$ name $>$ () is not defined! \\
\hline 033 & Error: Quantity $<$ name $>$ in function $<$ name $>$ () is not defined as parameter or global! \\
\hline 034 & Error: Unknown command line parameter name <name>! \\
\hline 035 & Error: Quantity <name>, parameter <param>, value <value> is not an integer! \\
\hline 036 & Error: Quantity <name>, parameter <param>, parameter unknown in <value $>$ ! \\
\hline 037 & $\begin{array}{l}\text { Error: Quantity <name>, parameter <param>, \%-character is not supported } \\
(<\text { value }>) \text { ! }\end{array}$ \\
\hline 038 & Error: Quantity <name>, parameter <param>, syntax error in <value>! \\
\hline 039 & Error: Quantity name <name> is a predefined function! \\
\hline 040 & Error: Quantity name <name> is a predefined symbol! \\
\hline 041 & Error: Quantity name <name> is a reserved name! \\
\hline 042 & Error: Quantity name <name> is the name of a user defined function! \\
\hline 043 & Error: Parameter name <name> is a predefined function! \\
\hline 044 & Error: Parameter name <name> is a reserved name! \\
\hline 045 & $\begin{array}{l}\text { Error: Quantity <name }>\text { in }<\text { Discrete }>\text { or }<\text { Import }>\text { element is unknown in the } \\
\text { bin-file }<\text { filename }>\text { ! }\end{array}$ \\
\hline 046 & Error: Index unknown! \\
\hline 047 & Error: Result name <name> is used twice in result section! \\
\hline
\end{tabular}




\begin{tabular}{|c|c|}
\hline \multicolumn{2}{|r|}{ Program exit codes } \\
\hline Exit Code & Error message \\
\hline 048 & Error: No result defined in source file! \\
\hline 049 & Error: Unknown symbol <name> in parameter value $\langle$ value $>$ ! \\
\hline 050 & Error: Unknown symbol in parameter value <value $>$ ! \\
\hline 051 & Error: Double assignment for $\mathrm{r}(\langle$ name $\rangle,\langle$ name $\rangle)$ : $\langle$ value $\rangle$ and $\langle$ value $\rangle$ ! \\
\hline 052 & Error: A near correlation matrix could not be found in <runs> runs! \\
\hline 053 & Error: No. of Runs in bin-file is zero! \\
\hline 054 & Error: Bin-file format $<$ format-id $>$ not supported! \\
\hline 055 & Error: Bin-file $<$ filename $>$ is corrupted! \\
\hline 056 & Error: Less than <value $>\%$ of the simulated results are valid! \\
\hline 057 & Error: The constrain for <name>: <expression> invalidates all data! \\
\hline 058 & Error: Output File Definition not found (<filename $\rangle) !$ \\
\hline 059 & Error: Constrain for <name>: <expression> cannot be evaluated! \\
\hline 060 & Error: Unknown command line parameter argument <argument>! \\
\hline 061 & Error: Parameter <param> in section <Simulation> is unknown! \\
\hline 062 & Error: Loop increment (Step) must be non-zero! \\
\hline 063 & Error: Parameter <param> in section <Loop> is unknown! \\
\hline 064 & Error: Error in <element> name: <text $>$ ! \\
\hline 065 & Error: quantile estimation method is unknown for $-\mathrm{pq}=\langle\mathrm{value}\rangle$ ! \\
\hline 066 & Error: The sub-block size -sbs=<value $>$ must be a multiple of 10 ! \\
\hline 067 & $\begin{array}{l}\text { Error: The block size -bs }=<\text { value }>\text { must be a multiple of the sub-block size } \\
- \text { sbs }=<\text { value }>\text { ! }\end{array}$ \\
\hline 068 & Error: Result data entry '<name>' unknown in ofd-file! \\
\hline 069 & Error: Result data entry '<name>' unknown in vfd-file! \\
\hline 254 & Error: Internal, Matrix structure does not match! \\
\hline 255 & Error: <error message>! \\
\hline
\end{tabular}

